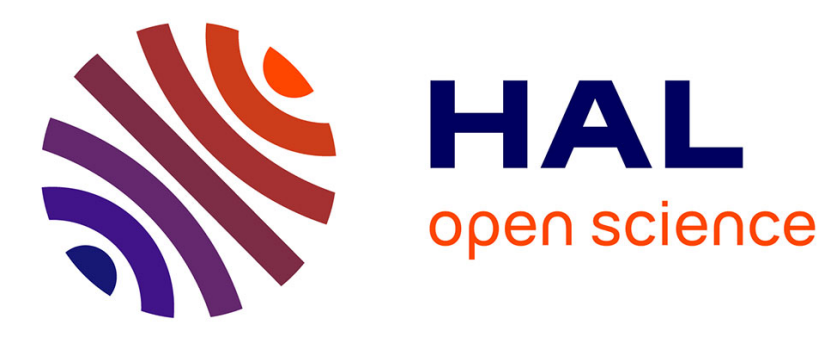

\title{
Modeling of a non-local stimulus for bone remodeling process under cyclic load: Application to a dental implant using a bioresorbable porous material
}

\author{
Ivan Giorgio, Ugo Andreaus, Daria Scerrato, Piero Braidotti
}

\section{To cite this version:}

Ivan Giorgio, Ugo Andreaus, Daria Scerrato, Piero Braidotti. Modeling of a non-local stimulus for bone remodeling process under cyclic load: Application to a dental implant using a bioresorbable porous material. 2016. hal-01306436

\author{
HAL Id: hal-01306436 \\ https://hal.science/hal-01306436
}

Preprint submitted on 23 Apr 2016

HAL is a multi-disciplinary open access archive for the deposit and dissemination of scientific research documents, whether they are published or not. The documents may come from teaching and research institutions in France or abroad, or from public or private research centers.
L'archive ouverte pluridisciplinaire HAL, est destinée au dépôt et à la diffusion de documents scientifiques de niveau recherche, publiés ou non, émanant des établissements d'enseignement et de recherche français ou étrangers, des laboratoires publics ou privés. 


\title{
Modeling of a non-local stimulus for bone remodeling process under cyclic load: Application to a dental implant using a bioresorbable porous material
}

\author{
Ivan Giorgio, Ugo Andreaus, Daria Scerrato, Piero Braidotti
}

I. Giorgio (Corresponding author), U. Andreaus - DeP. of Structural And Geotechnical Engineering, Università di Roma La Sapienza, 18 Via Eudossiana, 00184 - Rome, Italy

E-mail address of corresponding author: ivan.giorgio@uniroma1.it

I. Giorgio, D. Scerrato, P. Braidotti - Int. Research Center for the Mathematics and Mechanics of Complex Systems MeMoCS, Università dell'Aquila, 04012 - Cisterna di Latina, Italy

P. Braidotti - Dipartimento di Chirurgia Pietro Valdoni - Orthopedic Trauma Unit, Università di Roma La Sapienza, 5 Piazza Aldo Moro, 00161 - Rome, Italy

\begin{abstract}
In this paper, a numerical method based on finite elements is used to study the phenomena of resorption and growth of bone tissue and resorption of the biomaterial in the neighborhood of a dental implant fixture of the type IntraMobil Zylinder (IMZ). The mechanical stimulus that drives these processes is a linear combination of strain energy and viscous dissipation. To simulate the implant, an axisymmetric model has been used from the point of view of the geometry; the material behavior is described in the poro-visco-elastic frame. The external action is represented by a load variable with sinusoidal law characterized by different frequencies. Investigated aspects are the influence of the load frequency and of the lazy zone on the remodeling process.
\end{abstract}

Keywords: Dental implant, cycle load, resorbable biomaterial, dissipation, visco-poro-elastic material, bone remodeling

\section{Introduction}

Dental implants are artificial systems usually consisting of an endosteal component, which is completely implanted in the mandible or jawbone and of an abutment which connects the endosseous component with the oral cavity, in order to replace one or more missing teeth [1]. The most recent experimental researches have been aimed at the study of the response of bone tissue following the insertion of an implant. In particular, researchers tried to make functional and structural interfaces between implant and bone and, then, to improve the process of osseointegration through surface treatments and design optimization of 


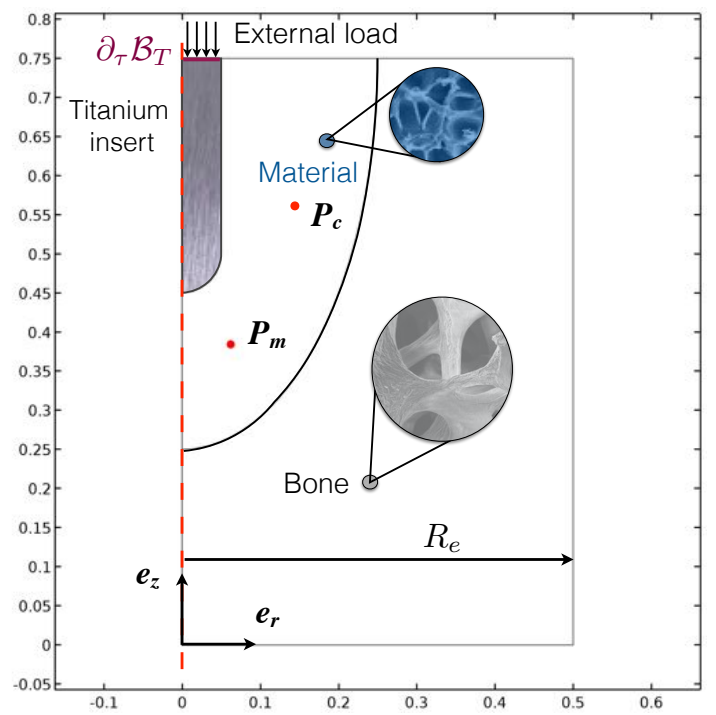

Figure 1: Initial arrangement of sample under study.

the system [2-4]. The reaction of the bone tissue, in fact, depends on chemical properties of the materials used and on the ability of the implant to induce a mechanical state of stress in the bone tissue that can promote osteogenesis processes. Research, today, follows two main routes: i) clinical trial designed to evaluate the incidence of known failure mechanisms of the implant, and ii) preclinical study that allows to conduct a preliminary analysis of the system. This work follows the latter route: in fact, the use of numerical simulation based on the finite element method appears advantageous in terms of time and costs compared to carrying out experiments in vivo or in vitro on dental implants. In addition, it seems useful to guide the clinical trial with a gallery of examples obtained by numerical studies. In relation to issues of possible failure in the medium to long term, this paper is interested in the study of the remodeling of bone in the neighborhood of the metal stem as its resorption may cause disconnection of the fixture from the host tissue and therefore implant mobilization. The issues are addressed with the use of computational tools and are intended to define design criteria to increase the average life of an implant. In particular, in this paper, we numerically study an implant model characterized by axial symmetry and composed of fixture, trabecular bone tissue and porous biomaterial. It allows to determine the evolution of the states of deformation, stress, energy and dissipation that are originated in the compound. The geometry of the device that inspired this research is the one of the IntraMobil Zylinder (IMZ) and the Bio-Vent ${ }^{\circledR}$ implant [5-7]. They are particularly suitable for soft and atrophic bone tissue, and are both characterized by coated cylinder implant designed for the mandibular jaw; the IMZ implant is coated with titanium plasma spray, the Bio-Vent implant is coated with hydroxyapatite. 


\section{Modeling}

A dental implant consists of a titanium cylinder or screw, between 8 and $16 \mathrm{~mm}$ long, inserted in the jaw into a predisposed bony socket and acts as a replacement root for supporting a false tooth. Specifically, it is made of a titanium alloy, a material that is known to be compatible with biological tissues and is able to bond with adjacent bone during the healing process. Indeed at this stage, the titanium surface of the implant fuses with the surrounding bone, in a process known as osseointegration. Only after this stage, which can take about 3-6 months, the implant is stable enough to support a new tooth. The titanium alloy presents also advantageous mechanical properties, namely, strength, endurance and good fatigue strength. In addition, this alloy can be fabricated with high precision to provide a wide variety of implants taking into account dimensions and state of health of bones and gums of a broad range of patients to ensure an optimal implant stability. Indeed, the problem of buckling due to the architecture of trabecular bone and the interaction with the graft under critical applied loads can occur. Possible strong problem singularities and instabilities can be regularized using methods described in the literature [8-12].

Often there is a need to increase the quantity of bone at the jawbone site with a graft to allow an optimal connection of the implant. The reason of this might be, for instance a lack of bone at that site due to a long-term missing tooth, a periodontal disease and aging or injury. In these cases, it is possible to use a synthetic bone substitute (resorbable alloplast) that acts as a sort of scaffold for the formation of new bone from the surrounding region and may be replaced, in part or completely, by natural bone.

In this paper, we consider a simple geometry to describe the interaction between a titanium insert, a bio-resorbable graft and the bone. In detail, we take into account a cylindrical region inside the jawbone which hosts the graft, shaped according to a half-ellipsoid, and the titanium insert (see Fig. 1). Cylindrical coordinates are the natural choice for this system exhibiting axial symmetry. Therefore, we introduce the usual coordinate for this reference frame characterized by the unit basis vectors $\left(\boldsymbol{e}_{\mathrm{r}}, \boldsymbol{e}_{\theta}, \boldsymbol{e}_{\mathrm{z}}\right)$. Following a Lagrangian description we identify the constituent particles of the system by their position in a geometric configuration taken as a reference and denoted with $\mathcal{C}^{*}$, that is to say by the variable vector $\boldsymbol{X}$ whose coordinates in the adopted reference frame are $(R, \Theta, Z)$. Then, we express the value of any physical value in the current configuration, i.e., $\mathcal{C}^{t}$, depending on the particle to which it is related and the current time, that is to say depending on variables $\boldsymbol{X}$ and $t$. In particular, a reference configuration is chosen such that the system is free-stress. However, since the system under study is characterized by a mass variable in time, the material properties of the reference configuration are not fixed, but evolve as the mass is varying. In addition, it is worth noting that the time scale of biological phenomena, namely, the evolution of the mass due to the remodeling process, is very slow compared to the scale characterizing the mechanical behavior and, therefore, the biological evolution of the system can be assumed not to entail the occurrence of any inertial effect due to mass variation.

By assuming for axisymmetric reasons that all the considered fields are invariant under rotations, the position vector $\boldsymbol{x}=(r, 0, z)$ of the particle located in $\mathcal{C}^{*}$ at position $\boldsymbol{X}$ is given by

$$
\boldsymbol{x}=r \boldsymbol{e}_{\mathrm{r}}+z \boldsymbol{e}_{\mathrm{z}}=\chi(R, Z, t)=R \boldsymbol{e}_{\mathrm{r}}+Z \boldsymbol{e}_{\mathrm{z}}+\boldsymbol{u}(R, Z, t)
$$

where the displacement $\boldsymbol{u}$ of the particle located in $\mathcal{C}^{*}$ at position $\boldsymbol{X}$ is obtained by

$$
\boldsymbol{u}(R, Z, t)=u_{\mathrm{r}}(R, Z, t) \boldsymbol{e}_{\mathrm{r}}+u_{\mathrm{z}}(R, Z, t) \boldsymbol{e}_{\mathrm{z}}
$$


We assume the transformation of the medium between the configurations $\mathcal{C}^{*}$ and $\mathcal{C}^{t}$ to be infinitesimal, namely, $\|\nabla \boldsymbol{u}(\boldsymbol{X}, t)\| \ll 1$, and therefore the Green-Lagrange strain tensor is reduced to the linearized strain tensor $\boldsymbol{E}$ that in cylindrical coordinates is expressed by the non-null components as

$$
E_{\mathrm{rr}}=\frac{\partial u_{\mathrm{r}}}{\partial R}, \quad E_{\mathrm{zz}}=\frac{\partial u_{\mathrm{z}}}{\partial Z}, \quad E_{\mathrm{rz}}=\frac{1}{2}\left(\frac{\partial u_{\mathrm{r}}}{\partial Z}+\frac{\partial u_{\mathrm{z}}}{\partial R}\right), \quad E_{\theta \theta}=\frac{u_{\mathrm{r}}}{R}
$$

To properly describe the behavior of the porous materials involved in this system, namely, living bone and artificial bio-resorbable material, we consider a solid mixture composed of two phases (see e.g. [13-15]), endowed with a microstructure that takes the presence of connected pores into account. As a result, in the framework of micro-morphic continua (see e.g. [16-19]), we introduce another kinematic descriptor able to characterize the mechanical response of pores inside the solid matrices considered (see [20-23] and for further developments [24-31]): the change of the Lagrangian porosity $\zeta$, i.e. the change of the effective volume of the fluid content per unit volume of the body, or mathematically

$$
\zeta(R, Z, t)=\phi(\chi(R, Z, t), t)-\phi^{*}(R, Z, t)
$$

where $\phi^{* 1}$ stands for the porosity in the reference configuration and $\phi$ is the Lagrangian porosity. The last can be expressed in terms of volume fraction of bone and artificial material, $\varsigma_{\mathrm{b}}$ and $\varsigma_{\mathrm{m}}$ respectively, as

$$
\phi=1-\left(\varsigma_{\mathrm{b}}+\varsigma_{\mathrm{m}}\right)=1-\left(\frac{\rho_{\mathrm{b}}}{\hat{\rho}_{\mathrm{b}}}+\frac{\rho_{\mathrm{m}}}{\hat{\rho}_{\mathrm{m}}}\right)
$$

where $\rho_{\mathrm{b}}, \rho_{\mathrm{m}}$ are the apparent mass densities of the two phases of the mixture, and $\hat{\rho}_{\mathrm{b}}, \hat{\rho}_{\mathrm{m}}$ are the true mass densities (with self-explanatory indexes). This kind of approach indeed aims to incorporate microscale effects, even if complex, into a continuum description (see e.g. [13,32-36] for applications to growth).

At the initial stage, the porous system is made of two distinct regions in which each of the phases, the artificial bio-resorbable material and the living bone, are separately present (see Fig. 1); it is only during the bone remodeling, in the process called osteoconduction, that new bone grows from the surrounding bone in the material region and therefore this zone exhibits both phases of the mixture considered. It is noted that the typical tools of the study of the phase transition can also be used in this context to model the rise of a new region in which both constituents coexist (see e.g. [37-40]).

Following the same approach as proposed in [41], we assume the hypothesis of isotropic and inhomogeneous material; moreover, denoting respectively by $\rho_{\mathrm{b}}^{*}$ and $\rho_{\mathrm{m}}^{*}$ the mass densities of bone and of bio-resorbable material in the reference configuration, the stored energy density $\mathscr{E}\left(\boldsymbol{E}, \zeta ; \rho_{\mathrm{b}}^{*}, \rho_{\mathrm{m}}^{*}\right)$ associated with strain tensor $\boldsymbol{E}$ and fluid volume distortion from the reference configuration $\zeta$ can be represented as

$$
\mathscr{E}=\mathcal{E}_{s}\left(\boldsymbol{E} ; \rho_{\mathrm{b}}^{*}, \rho_{\mathrm{m}}^{*}\right)+\frac{1}{2} K_{1}\left(\rho_{\mathrm{b}}^{*}, \rho_{\mathrm{m}}^{*}\right) \zeta^{2}+\frac{1}{2} K_{2}\|\nabla \zeta\|^{2}-K_{3}\left(\rho_{\mathrm{b}}^{*}, \rho_{\mathrm{m}}^{*}\right) \zeta \operatorname{tr} \boldsymbol{E}
$$

where $\mathcal{E}_{s}=\frac{1}{2} T_{\mathrm{ij}}^{e l} E_{\mathrm{ij}}$ is the bulk strain-dependent energy density, and $T_{\mathrm{ij}}^{e l}$ is the second Piola stress tensor given by the constitutive relation:

$$
T_{\mathrm{ij}}^{e l}=\lambda\left(\rho_{\mathrm{b}}^{*}, \rho_{\mathrm{m}}^{*}\right) \operatorname{tr} \boldsymbol{E} \delta_{\mathrm{ij}}+2 \mu\left(\rho_{\mathrm{b}}^{*}, \rho_{\mathrm{m}}^{*}\right) E_{\mathrm{ij}}
$$

\footnotetext{
${ }^{1}$ From this point on, the superscript ${ }^{*}$ denotes all quantities in the reference configuration.
} 
being $\lambda$ and $\mu$ the Lamé parameters and where we associate the values taken by the subscripts $\mathrm{i}$ and $\mathrm{j}$ ranging over the set $\{1,2,3\}$, in the given order, to the directions of the reference frame $r, \theta, z$ in order to use the summation convention. Eq. (7) can be written in terms of Young's modulus, $Y$, and Poisson's ratio, $\nu$, by recalling the following relationships:

$$
\lambda=\frac{Y\left(\rho_{\mathrm{b}}^{*}, \rho_{\mathrm{m}}^{*}\right) \nu}{(1-2 \nu)(1+\nu)} \quad \mu=\frac{Y\left(\rho_{\mathrm{b}}^{*}, \rho_{\mathrm{m}}^{*}\right)}{2(1+\nu)}
$$

As a first approximation, Poisson's ratio is assumed to be constant, while we hypothesize that Young's modulus changes with the bone remodeling according to the power-law:

$$
Y=Y_{\mathrm{b}}^{\operatorname{Max}}\left(\varsigma_{\mathrm{b}}^{*}\right)^{\beta_{\mathrm{b}}}+Y_{\mathrm{m}}^{\operatorname{Max}}\left(\varsigma_{\mathrm{m}}^{*}\right)^{\beta_{\mathrm{m}}}
$$

where $Y_{\mathrm{b}}^{\mathrm{Max}}$ and $Y_{\mathrm{m}}^{\mathrm{Max}}$ are the maximal elastic moduli and the exponents $\beta_{\mathrm{b}}, \beta_{\mathrm{m}}$ are constants.

The coefficient $K_{1}$ which can be interpreted as a coefficient of compressibility related to the fluid phase inside pores, i.e. the bone marrow, is assumed to depend on the reference porosity as follows:

$$
K_{1}=\left(\frac{\phi^{*}}{K_{\mathrm{f}}}+\frac{\left(\alpha_{B}-\phi^{*}\right)\left(1-\alpha_{B}\right)}{K_{\mathrm{dr}}}\right)^{-1}
$$

where $K_{\mathrm{f}}$ is the marrow modulus, $K_{\mathrm{dr}}=Y /(3(1-2 \nu))$ is the drained bulk modulus of the porous matrix and $\alpha_{B}$ is the Biot-Willis coefficient that satisfies the inequality $\phi^{*} \leqslant \alpha_{B} \leqslant 1$. Moreover, the trace of the small strain tensor $\boldsymbol{E}$

$$
\operatorname{tr} \boldsymbol{E}=\frac{\partial u_{\mathrm{r}}}{\partial R}+\frac{u_{\mathrm{r}}}{R}+\frac{\partial u_{\mathrm{z}}}{\partial Z}
$$

is introduced to describe the interaction between the solid and the microstructure.

The gradient of the change of porosity, because of the axial symmetry, is given by

$$
\nabla \zeta(R, Z, t)=\frac{\partial \zeta}{\partial R} \boldsymbol{e}_{\mathrm{r}}+\frac{\partial \zeta}{\partial Z} \boldsymbol{e}_{\mathrm{z}}
$$

and is used as an application of a second gradient theory - Cahn-Hilliard fluids - to take capillary interaction phenomena among neighbouring pores into account [42]. The material parameter $K_{2}$ that appears in Eq. (6) can be thus interpreted as a stiffness related to the influence of capillarity governed by the $\nabla \zeta$. Higher order theories of deformation can also be usefully employed to model damage evolution of bone (see e.g. [43-49]) which may be relevant in the remodeling process [50].

The material parameter $K_{3}$ introduced in Eq. (6) can be physically interpreted as the coupling between microstructure and solid bulk [41] and is evaluated according to the relation:

$$
K_{3}=\sqrt{\hat{g}\left(\phi^{*}\right) \lambda K_{1}}
$$

where the monotonic function $\hat{g}\left(\phi^{*}\right)$ takes non-negative value less then one (see Fig. 2) and is designed to emphasize the effect of pores when the level of porosity is high and to understate this effect when porosity decreases according to:

$$
\hat{g}\left(\phi^{*}\right)=\frac{A_{k_{3}}}{\pi}\left\{\operatorname{atan}\left[s_{k_{3}}\left(\phi^{*}-\frac{1}{2}\right)\right]+\operatorname{atan}\left(\frac{s_{k_{3}}}{2}\right)\right\} \quad 0<A_{k_{3}} \leqslant 1
$$




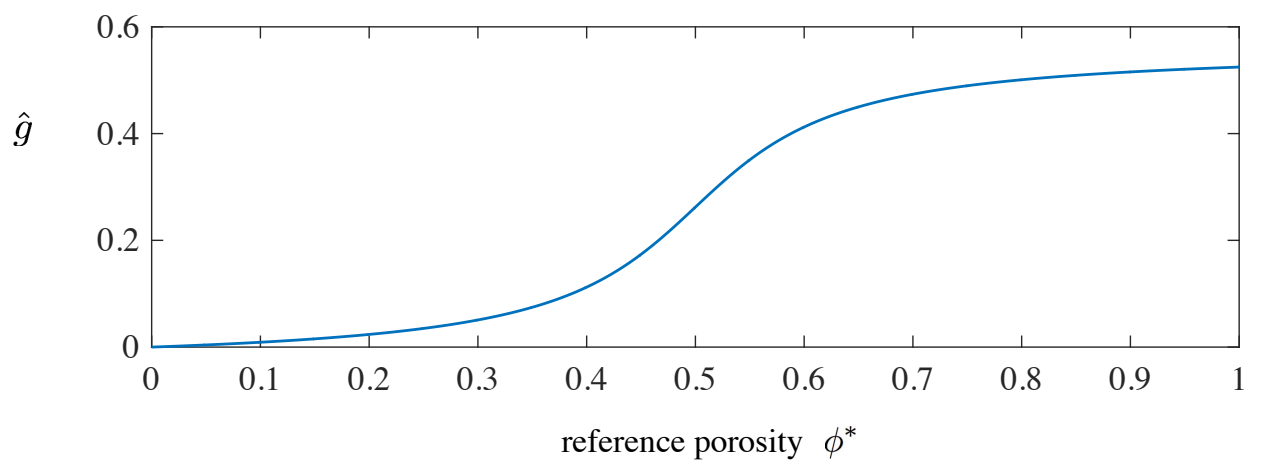

Figure 2: Function $\hat{g}\left(\phi^{*}\right)$.

where $A_{k_{3}}$ and $s_{k_{3}}$ are shape coefficients that can be chosen to characterize the coupling law adopted. Specifically, the coefficient $A_{k_{3}}$ modulates the amplitude of the coupling law Eq. (14) while $s_{k_{3}}$ governs the slope of the above mentioned law. Recalling that the material parameter $K_{3}$ governs the coupling between the variables of the solid bulk and those of the microstructure, the coupling law Eq. (14) has been designed in order to i) penalize the coupling when the porosity tends to zero, and then the global behavior tends to the one of the solid bulk, and ii) exalt the coupling when the porosity tends to one, and then the role of the microstructure becomes increasingly more important.

In order to guarantee the positive definiteness of the stored energy density (6), the following inequalities which involve the material parameters above defined should be satisfied as stated in [20]:

$$
\mu \geqslant 0, \quad \kappa=\lambda+2 / 3 \mu \geqslant 0, \quad K_{1} \geqslant 0, \quad K_{2} \geqslant 0, \quad \kappa K_{1} \geqslant 4 K_{3}^{2}
$$

Finally, interface conditions for the variable $\zeta$ can be formulated by adding to the energy density of Eq. (6) a boundary extra term:

$$
\mathscr{E}_{\text {int }}=\frac{1}{2} K_{4}\left(\zeta^{+}-\zeta^{-}\right)^{2}=\frac{1}{2} K_{4} \llbracket \zeta \rrbracket^{2} \quad \text { with } K_{4} \geqslant 0
$$

which accounts for the contribution of the interface, $\partial \mathcal{B}_{\text {int }}$, between bone and material regions. Therefore, the material parameter $K_{4}$ plays the role of a stiffness that rules the interaction between the two materials in terms of the jump of $\zeta$. For the sake of simplicity, the coefficients $K_{2}$ and $K_{4}$ are assumed to be constant.

Some features suggests the presence of viscous damping in bone [51]: total deformation under a constant stress was not attained instantaneously, i.e, bone has a retardation time; an hysteresis loop arises from loading-unloading cyclic tests; and so forth. Therefore, according to the Kelvin-Voigt formulation, viscous damping can be added to the material model of the porous mixture by means of an extra stress $T_{\mathrm{ij}}^{v}$ related to the rate of elastic strain of the material

$$
T_{\mathrm{ij}}^{v}(\dot{\boldsymbol{E}})=2 \mu^{v}\left(\dot{E}_{\mathrm{ij}}-\frac{1}{3} \dot{E}_{\mathrm{kk}} \delta_{\mathrm{ij}}\right)+\kappa^{v} \dot{E}_{\mathrm{kk}} \delta_{\mathrm{ij}}
$$


where $\kappa^{v}$ and $\mu^{v}$ are the bulk and shear viscosity coefficients, respectively. As a result, the dissipated power is given by

$$
2 \mathcal{D}_{s}=T_{\mathrm{ij}}^{v} \dot{E}_{\mathrm{ij}}
$$

It is worth observing that the validity of this simple model, satisfactory for low physiological rate evolutions [52], becomes increasingly inadequate beyond the range of quasi-static loading which is herein considered (for more complex models see e.g. [53,54]).

Since the problem under study is time-dependent, but the evolution in time is very slow, a quasi-static formulation is assumed and then inertial effects are neglected. The numerical code used to calculate the configuration sequences of the considered system includes a formulation of the poro-viscoelastic problem linking external mechanical applied loads to the corresponding equilibrium deformation states. However, it is also required the knowledge of the evolution of the mass densities, from which the stiffness of the mixture, given by Eq. (9), can be evaluated at each step. The evolution problem involves only the equations for time variations of apparent mass densities of the living bone tissue and the bio-resorbable material. On the other hand, since time variations of mixture stiffness and variations of loads occur in a time scale which is much larger than the transient characteristic time leading to macroscopic mechanical equilibrium, the two problems, the mechanical and the evolutionary one, can be separately solved at each time step in sequence with an iterative scheme as time increases.

\subsection{Poro-viscoelastic formulation}

The mechanical governing equations of the graft/bone system can be deduced from the generalized principle of virtual work. Indeed, the total virtual work, including dissipative actions (see e.g. [55]), for any arbitrary virtual displacement $\boldsymbol{u}$ and for a virtual change of porosity $\zeta$ equal to zero is given by

$$
\begin{aligned}
-\int_{\mathcal{B}}\left[T_{\mathrm{ij}}^{e l} \delta E_{\mathrm{ij}}+K_{1} \zeta \delta \zeta+K_{2} \zeta_{\mathrm{i}} \delta \zeta_{, \mathrm{i}}-K_{3}\left(E_{\mathrm{ii}} \delta \zeta+\zeta \delta E_{\mathrm{ii}}\right)\right] \mathrm{d} \mathcal{V}-\int_{\mathcal{B}} T_{\mathrm{ij}}^{v} \delta E_{\mathrm{ij}} \mathrm{d} \mathcal{V} & \\
& -\int_{\partial \mathcal{B}_{\mathrm{int}}} K_{4} \llbracket \zeta \rrbracket \delta \llbracket \zeta \rrbracket \mathrm{d} \mathcal{S}+\int_{\partial_{\mathcal{\tau}} \mathcal{B}} \tau_{\mathrm{i}} \delta u_{\mathrm{i}} \mathrm{d} \mathcal{S}+\int_{\partial \mathcal{B}} \Xi \delta \zeta \mathrm{d} \mathcal{S}=0
\end{aligned}
$$

To solve our problem, we consider a mixed boundary value problem in which we impose the displacement components

$$
\begin{array}{ll}
u_{\mathrm{r}}\left(R_{e}, Z, t\right)=0 & \forall Z \text { and } t \\
u_{\mathrm{z}}(R, 0, t)=0 & \forall R \text { and } t
\end{array}
$$

being $R_{e}$ the external radius of the cylinder under study. The titanium stem (here modeled as a linear elastic solid) is loaded by an external pressure on its top, i.e. $\partial_{\tau} \mathcal{B}_{T}$ (see Fig. 1); in turn, the titanium insert applies a surface traction $\tau_{\mathrm{i}}$ on the boundary $\partial_{\tau} \mathcal{B}$ between the titanium stem itself and the mixture. Possibly a micro-structural action $\Xi$, related to the dilatant behavior of the porous mixture, can be applied on the boundary $\partial \mathcal{B}$. For further information about contact interactions in more general cases see for example $[56,57]$. Since inertia effects are neglected but the mechanical governing equation is a differential 
equation of first order in time for the presence of the dissipative actions, it is worth recalling that herein the initial conditions are reduced to

$$
\boldsymbol{u}(R, Z, 0)=0 \quad \forall R, Z
$$

\subsection{Evolution rules for the mass densities}

The evolution equations for apparent mass densities are assumed to be first order ordinary differential equations with respect to time. We also assume that the spatial gradient of mass densities does not affect the remodeling equations, and therefore we simply assume that the process of remodeling is non-local only as a function of the nature of the stimulus (herein it is obtained by an integral operator), and it does not depends on what is occurring outside the representative elementary volume by other non-local phenomena of pure mechanical nature. As a result, we assume that the total mass density rates depend on the stimulus and the porosity field as follows:

$$
\begin{cases}\dot{\rho}_{\mathrm{b}}^{*}=A_{\mathrm{b}}(S) H(\phi) & \text { with } 0<\rho_{\mathrm{b}}^{*} \leqslant \hat{\rho}_{\mathrm{b}} \\ \dot{\rho}_{\mathrm{m}}^{*}=A_{\mathrm{m}}(S) H(\phi) & \text { with } 0<\rho_{\mathrm{m}}^{*} \leqslant \rho_{\mathrm{m}}^{0}\end{cases}
$$

where $A_{\mathrm{b}}$ and $A_{\mathrm{m}}$ are piece-wise linear functions with different slopes for negative $\left(r_{\mathrm{b}}\right.$ and $\left.r_{\mathrm{m}}\right)$ and positive values of stimulus $\left(s_{\mathrm{b}}\right)$; moreover for the bio-resorbable material and positive values of the stimulus the function $A_{\mathrm{m}}$ vanishes, since synthesis of bio-resorbable material cannot occur. Indeed, the maximum value possible for $\rho_{\mathrm{m}}^{*}$ is its initial one, i.e. $\rho_{\mathrm{m}}^{0}$. Specifically, we have

$$
\begin{aligned}
& A_{\mathrm{b}}(S)= \begin{cases}s_{\mathrm{b}} S & \text { for } S \geq 0 \\
r_{\mathrm{b}} S & \text { for } S<0\end{cases} \\
& A_{\mathrm{m}}(S)= \begin{cases}0 & \text { for } S \geq 0 \\
r_{\mathrm{m}} S & \text { for } S<0\end{cases}
\end{aligned}
$$

The function $H$ (see Fig. 3) is evaluated as a normalized Specific Surface, i.e., the internal surface area per unit volume of whole bone [58]. Indeed, this function is related to the surface available for resorption or synthesis 'inside' the considered material particle which, in its turn, depends on the 'effective' porosity field [59].

To take the experimental evidence into account, we consider a so called 'lazy zone' for stimulus. Particularly, when the signal falls in a given range then the effect of the actor cells is not macroscopically appreciable. For this purpose, we introduce two activation thresholds, $P_{\text {ref }}^{\mathrm{s}}$ and $P_{\text {ref }}^{\mathrm{r}}$ ('s' stands for synthesis while ' $r$ ' means resorption), that define the width of this 'lazy zone' associated with a balanced biological equilibrium state and, therefore, the stimulus becomes:

$$
S(\boldsymbol{X}, t)= \begin{cases}P(\boldsymbol{X}, t)-P_{\mathrm{ref}}^{\mathrm{s}} & \text { for } P(\boldsymbol{X}, t)>P_{\mathrm{ref}}^{\mathrm{s}} \\ 0 & \text { for } P_{\mathrm{ref}}^{\mathrm{r}} \leqslant P(\boldsymbol{X}, t) \leqslant P_{\mathrm{ref}}^{\mathrm{s}} \\ P(\boldsymbol{X}, t)-P_{\mathrm{ref}}^{\mathrm{r}} & \text { for } P(\boldsymbol{X}, t)<P_{\mathrm{ref}}^{\mathrm{r}}\end{cases}
$$




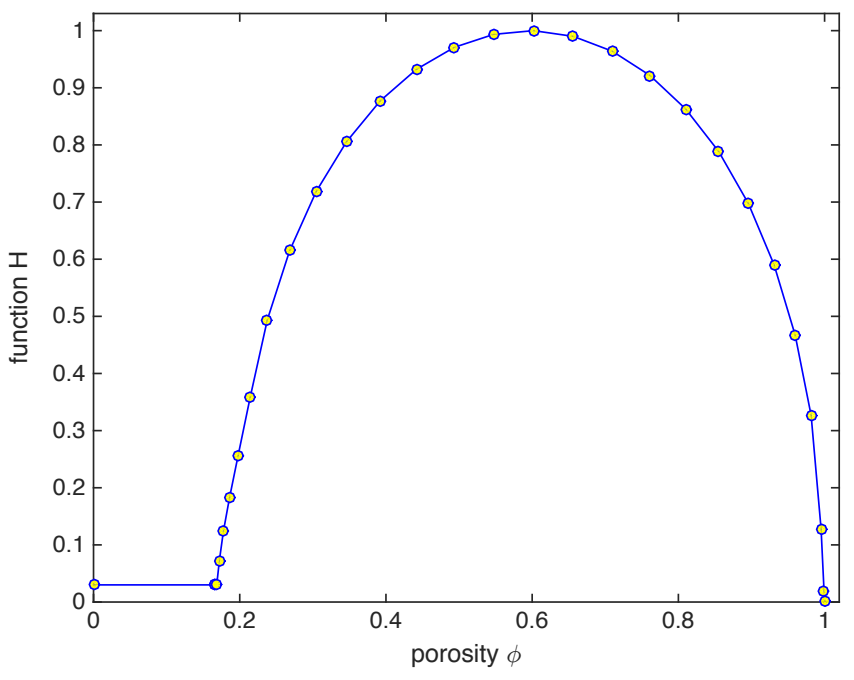

Figure 3: Function $H$.

where $P(\boldsymbol{X}, t)$ is

$$
P(\boldsymbol{X}, t)=\frac{\int_{\mathcal{B}}\left[a \mathcal{E}_{s}\left(\boldsymbol{X}_{0}, t\right)+b \mathcal{D}_{s}\left(\boldsymbol{X}_{0}, t\right)\right] \varpi\left[\rho_{\mathrm{b}}\left(\boldsymbol{X}_{0}, t\right)\right] e^{-\frac{\left\|\boldsymbol{X}-\boldsymbol{X}_{0}\right\|^{2}}{2 D^{2}}} \mathrm{~d} \boldsymbol{X}_{0}}{\int_{\mathcal{B}} e^{-\frac{\left\|\boldsymbol{X}-\boldsymbol{X}_{0}\right\|^{2}}{2 D^{2}}} \mathrm{~d} \boldsymbol{X}_{0}}
$$

We assume that the signal stemming from sensor cells in a given material particle of the bone/bio-material mixture is instantaneously transmitted, i.e., the transmission time scale is negligible compared to the characteristic time of the remodeling phenomena, and that its intensity decays exponentially with the distance from it [59-61]. Besides, to emulate the non-local behavior due to the sensor cell network, we express the stimulus as a spatially averaged signal over a spherical region of radius $D$ which represents the range of action of sensor cells [62]. The function $\varpi$ is assumed to be:

$$
\varpi\left(\rho_{\mathrm{b}}^{*}\right)=\eta \tanh \left(\xi \rho_{\mathrm{b}}^{*}\right) \quad 0<\eta \leqslant 1
$$

and it can be interpreted, in the framework of the feedback control theory [63], as a gain for the actual mechanical stimulus, i.e., 'the actuating signal', that takes the real activities of the osteocytes into account [58].

Several stimulus models have been proposed in literature to match experimental observations. All these models are characterized by a particular description of the stimulus; in some of them it is based on the strain energy density [59,60,64], on a strain-damage energy density [65], on the adaptation of the elastic modulus of the bone coupled directly to deviations of the strain tensor [66], while others propose a creepfatigue cumulative damage model $[67,68]$, or a global optimal criterion for remodeling [69-74]. In this 
paper, we propose for bone adaptation the use of a linear combination of the strain-dependent energy, $\mathcal{E}_{s}$, and the dissipation power, $\mathcal{D}_{s}$, in order to predict two important features of the remodeling phenomenon: the bone capability to control its mass and stiffness directly related to static mechanical demands [59,64] for the function of load-carrying capacity, and the effects of the frequency of applied loads $[75,76]$ for the function of damping and shock absorption.

As a note, herein, we consider internal remodeling as being the resorption or deposition of bone material, accompanied by the removal and densification of the architecture of bone, but without any change in the overall shape $[41,59,77,78]$. An external or surface modeling is not studied. Such a kind of remodeling causes a change of the external shape of the overall bone structure, and occurs by the resorption or deposition of bone material on the surfaces (see e.g. [32,33]).

The bio-material which we assume to use as a graft is absolutely not active in both sensing and actuating process. However, one could conceive a 'smart' prosthesis having much more effective performances by using the concept developed in [79-83] or some new metamaterials [84] which exhibit interesting features such as lightness and resistance to damage as proposed in [85-88].

\section{$3 \quad$ Numerical simulations}

In order to test the predictive capabilities of the proposed model, we performed numerical simulations, using the commercial software COMSOL Multiphysics ${ }^{\circledR}$, on a three-dimensional cylindrical sample of height $h_{b}=10 \mathrm{~mm}$ and radius $R_{e}=2 h_{b} / 3$. The sample is made of a piece of jawbone, a bio-material graft and a titanium insert as sketched in Fig. 1.

Initially, the mass density distributions of the bone and of the graft material are taken spatially uniform and segregated in two distinct zones, $\mathcal{C}_{b}$ and $\mathcal{C}_{m}$ respectively, to deal with a simple but representative case for a dental implant. Hence, initial conditions for bone mass density, $\rho_{\mathrm{b}}^{*}$, and bio-material density, $\rho_{\mathrm{m}}^{*}$, are

$$
\begin{cases}\rho_{\mathrm{b}}^{*}(\boldsymbol{X}, 0)=\rho_{\mathrm{b}}^{0}=\hat{\rho}_{\mathrm{b}} / 2 & \forall \boldsymbol{X} \in \mathcal{C}_{b} \\ \rho_{\mathrm{m}}^{*}(\boldsymbol{X}, 0)=\rho_{\mathrm{m}}^{0}=\hat{\rho}_{\mathrm{m}} / 2 & \forall \boldsymbol{X} \in \mathcal{C}_{m}\end{cases}
$$

Herein, the problem under study is recast in a non-dimensional form by normalizing the stored elastic energy with respect to the maximal stiffness of bone $Y_{\mathrm{b}}^{\mathrm{Max}}=18 \mathrm{GPa}$ and the dissipated power by $Y_{\mathrm{b}}^{\mathrm{Max}} / t_{\mathrm{ref}}$, being $t_{\text {ref }}$ a reference time assumed equal to $7.2576 \times 10^{6} \mathrm{~s}$; the lengths are normalized with respect to the diameter of the sample, and the apparent mass densities with respect to the $\hat{\rho}_{\mathrm{b}}=1,800 \mathrm{~kg} / \mathrm{m}^{3}$, which is assumed equal to $\hat{\rho}_{\mathrm{m}}$. The non-dimensional quantities are denoted by a tilde, and can be summarized as:

$$
\begin{array}{ll}
\tilde{K}_{\mathrm{f}}=K_{\mathrm{f}} / Y_{\mathrm{b}}^{\mathrm{Max}}, & \tilde{K}_{2}=K_{2} /\left(Y_{\mathrm{b}}^{\operatorname{Max}} 4 R_{e}^{2}\right), \\
\tilde{\lambda}=\lambda / Y_{\mathrm{b}}^{\mathrm{Max}}, & \tilde{K}_{4}=K_{4} /\left(Y_{\mathrm{b}}^{\operatorname{Max}} 2 R_{e}\right), \\
\tilde{\mu}^{v}=\mu^{v} /\left(t_{\mathrm{ref}} Y_{\mathrm{b}}^{\mathrm{Max}}\right), & \tilde{\kappa}^{v}=\kappa^{v} /\left(t_{\mathrm{ref}} Y_{\mathrm{b}}^{\mathrm{Max}}\right)
\end{array}
$$

A cyclic compression load case is considered, in which an external force is applied on the titanium insert along its longitudinal axis; in particular, the force $\tau_{z}^{e x t}(t)=-F_{0}-F_{1} \sin (2 \pi f t)$ with a pre-load $F_{0}$ and a cyclic contribution of magnitude $F_{1}=F_{0} / 2$ and frequency $f=\{5,10,20\}$ cycles per unit of time is 
Table 1: Values of the macro-parameters used in numerical simulations.

\begin{tabular}{ccccccccc}
\hline$\tilde{Y}_{\mathrm{T}}$ & $\tilde{Y}_{\mathrm{b}}^{\operatorname{Max}}$ & $\tilde{Y}_{\mathrm{m}}^{\mathrm{Max}}$ & $\beta_{\mathrm{b}}=\beta_{\mathrm{m}}$ & $\tilde{\mu}^{v}$ & $\tilde{\kappa}^{v}$ & $\tilde{D}$ & $\eta$ & $\xi$ \\
\hline 6 & 1 & 1 & 1.5 & 0.1 & $1 / 6$ & $1 / 5$ & 0.1 & 10 \\
\hline
\end{tabular}

Table 2: Values of the micro-parameters used in numerical simulations.

\begin{tabular}{ccccc}
\hline$\tilde{K}_{\mathrm{f}}$ & $\tilde{K}_{2}$ & $A_{k 3}$ & $s_{k 3}$ & $\tilde{K}_{4}$ \\
\hline 0.1 & $1.0 \times 10^{-3}$ & 0.6 & 10 & 0.1 \\
\hline
\end{tabular}

employed. The non-dimensional value of the force is $\tilde{F}_{0}=0.00286$ obtained normalizing by the stiffness $Y_{\mathrm{b}}^{\mathrm{Max}}$. In absolute terms, the external force applied on the top of the fixture corresponds to a typical masticatory load of about $160 \mathrm{~N}$ on the abutment top. Young's modulus of titanium insert is assumed to be $Y_{\mathrm{T}}=108 \mathrm{GPa}$. The limit values that define the range of the 'lazy zone' are $\tilde{P}_{\text {ref }}^{\mathrm{r}}=1.63 \times 10^{-9}$ and $\tilde{P}_{\text {ref }}^{\mathrm{s}}=1.84 \times 10^{-9}$. The values of the parameters used in the performed numerical simulations are summarized in Tabs. $1-3$. Plots of some results relatively to the probe point $P_{m} \equiv\left(R_{e} / 8,10 / 13 R_{e}\right)$ in the time range considered for simulation are showed since this point is located in the most stressed area, and it involves the evolution of both components of the mixture.

As a side note, recent applications of the finite element method (as discussed in [89-99]) could be usefully employed in this context to enhance the possibilities to improve the formulation and thus to ensure a better fit for experimental evidence.

Figure 4 shows the reference porosity $\phi^{*}$ at the end of the process for different frequencies of loading. With reference to the region of the biomaterial, it is observed that for low frequencies, the process is slower and the biomaterial has more time to be reabsorbed. The area with high porosity (0.7 - blue zone) compared to initial value (0.5) is very wide at frequency of 5 cycles per unit of time and is gradually being reduced with increasing frequency; in this area the porosity of the mixture coincides with the one of the biomaterial; in the red area (which is the most stressed because it is below the implant) where the porosity decreases, there is growth of bone tissue and therefore the porosity is equal to the one of the mixture. For high frequencies, the growth of bone tissue invades the entire region of the biomaterial (red zone) and the

Table 3: Rates of resorption and synthesis.

\begin{tabular}{lll}
\hline$\tilde{s}_{\mathrm{b}}$ & $\tilde{r}_{\mathrm{b}}$ & $\tilde{r}_{\mathrm{m}}$ \\
\hline 12 & 10 & 15 \\
\hline
\end{tabular}




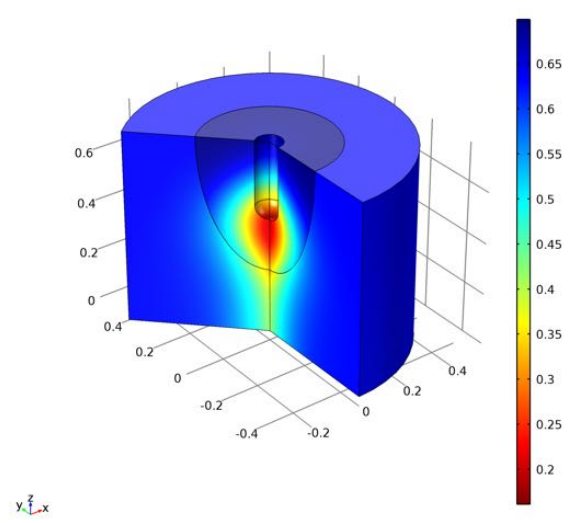

(a) frequency of 5 cycles per unit of time

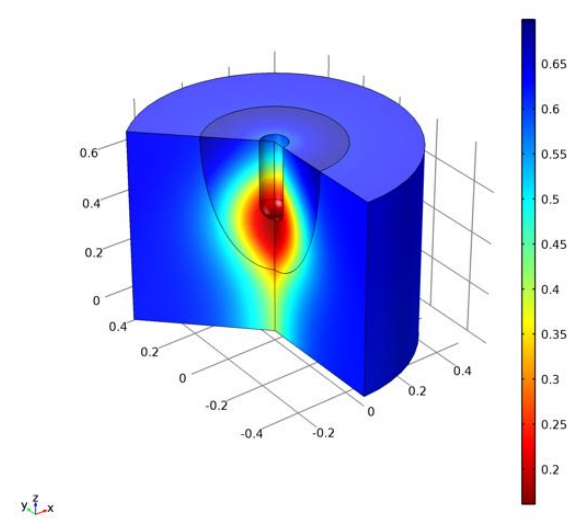

(b) frequency of 10 cycles per unit of time

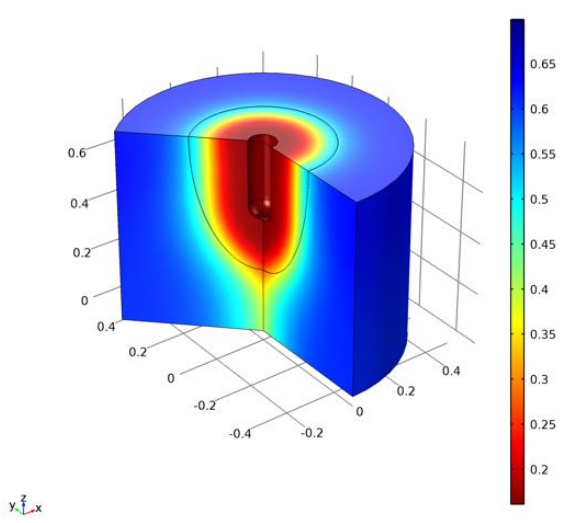

(c) frequency of 20 cycles per unit of time

Figure 4: Reference porosity $\phi^{*}$ at the end of the simulation.

minimum porosity $(0.2)$ is reached.

Figure 5 shows the final mass densities of biomaterial represented in terms of variables $R, Z$ at the end of the simulation for different frequencies. The figure indicates that in the region of the biomaterial (or at least in the more external discharged part) the reduction of the mass density of the material, in front of bone growth, decreases as the frequency increases (from 0.5 to 0.3 at 5 cycles per unit of time, 0.5 to 0.32 at 10 cycles per unit of time, and finally from 0.5 to 0.41 at 20 cycles per unit of time), because the bone tissue colonizes the material quicker as the frequency increases.

Figure 6 shows the change of the porosity $\zeta$ at the end of the process for different frequencies. The increase of frequency causes the mixture to exhibit a lower porous shrinkage leading the bone to grow faster and the pores to be filled quicker. Hence, the system will become more compact.

Figure 7 shows the time histories of the mass densities of bone (solid line) and material (dotted-dashed line) at the point $P_{m}$ probe for different frequencies. It is noted that while the mass density of the bone also grows significantly as the frequency increases, the mass density of the material remains almost constant and independent of frequency, as the bone is filling the voids of biomaterial.

Figure 8 shows the time histories of the change of porosity $\zeta$ at the probe point $P_{m}$. As the frequency increases the mass density of mixture grows and the porosity decreases (see Fig. 4), therefore the mixture becomes stiffer and hence the change of porosity $\zeta$ oscillates within an increasingly narrower band. The trend is clearly stabilizing towards the stationary state confirming the adequate choice of the analysis duration.

Figures 9 and 10 show the time histories of the elastic energy $\mathcal{E}_{s}$ and of the dissipation power $\mathcal{D}_{s}$ at the probe point $P_{m}$. It confirms the fact that, as the frequency increases, the mixture exhibits a less deformable and more dissipative behavior.

Figure 11 shows the energy dissipation loops for the three principal directions at the probe point $P_{c}$ 


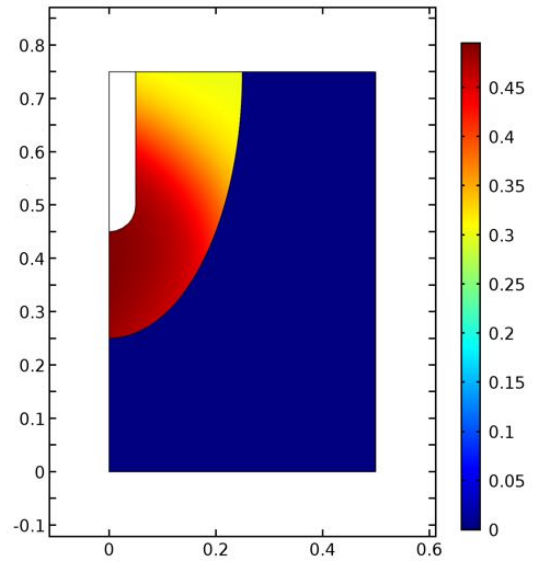

(a) frequency of 5 cycles per unit of time

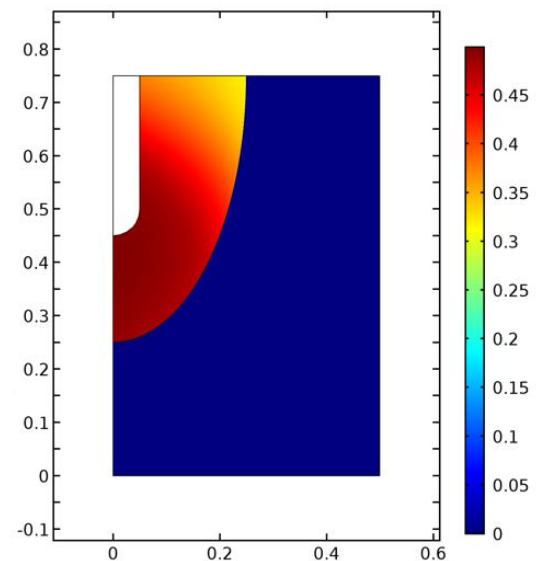

(b) frequency of 10 cycles per unit of time

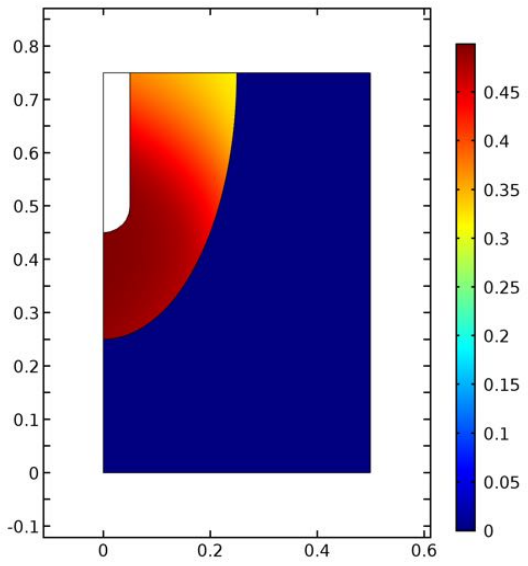

(c) frequency of 20 cycles per unit of time

Figure 5: Normalized mass density of bio-material at the end of the simulation.

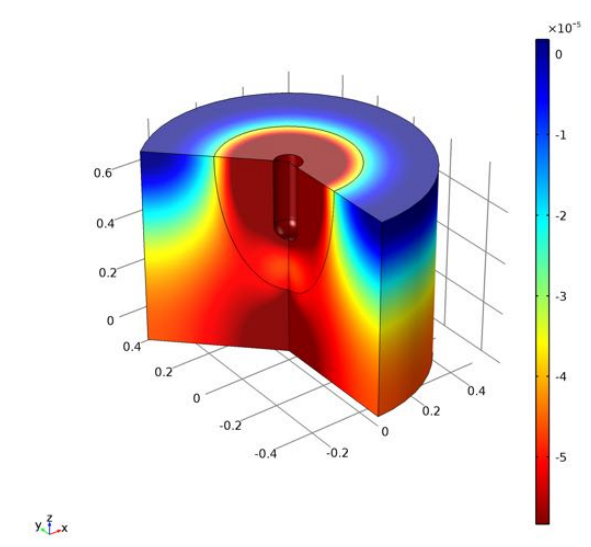

(a) frequency of 5 cycles per unit of time

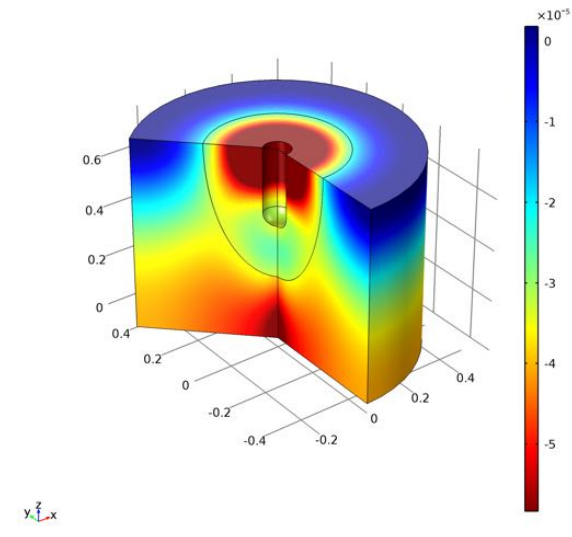

(b) frequency of 10 cycles per unit of time

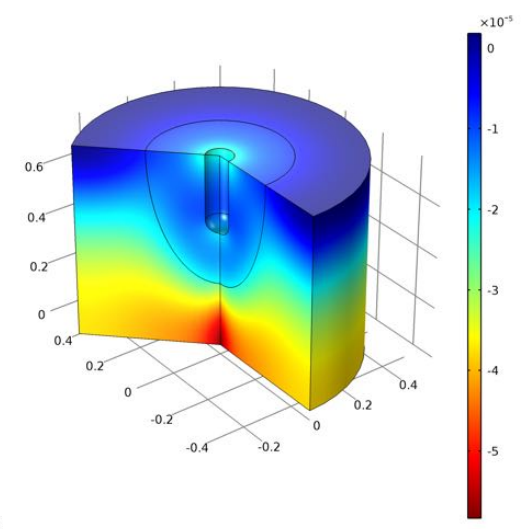

(c) frequency of 20 cycles per unit of time

Figure 6: Change of porosity $\zeta$ at the end of the simulation. 


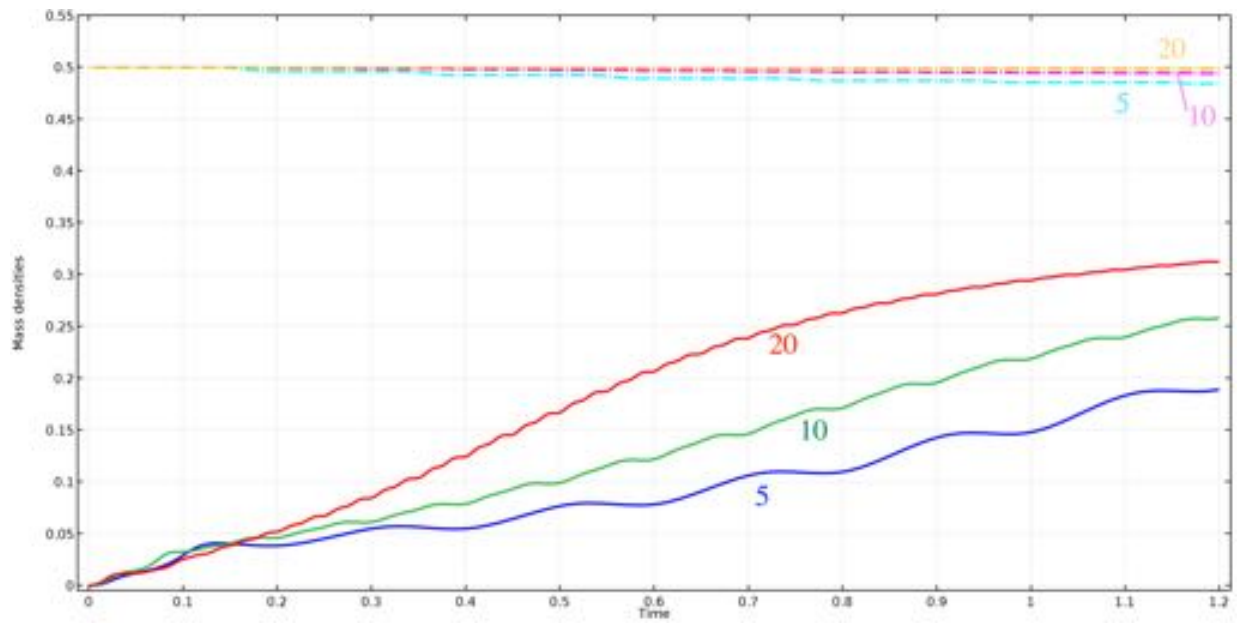

Figure 7: Mass density histories of bone (solid line) and of bio-resorbable material (dotted-dashed line) at the probe point $P_{m}$ for different load frequencies (5, 10 and 20 cycles per unit of time).

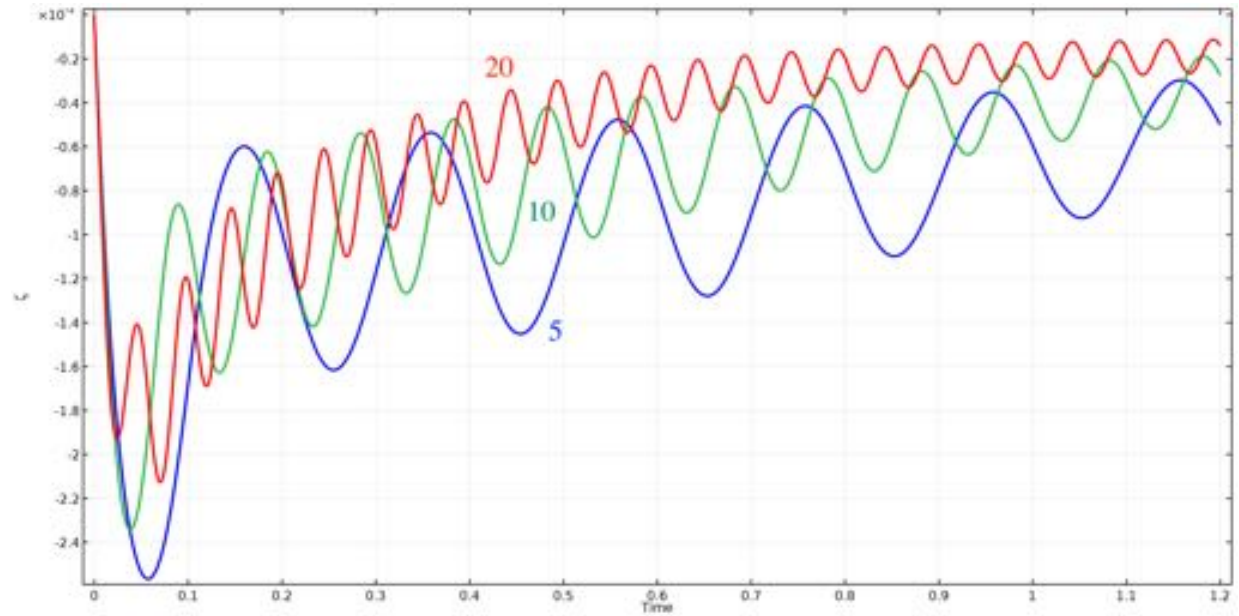

Figure 8: History of the change of the porosity $\zeta$ at the probe point $P_{m}$ for different load frequencies $(5$, 10 and 20 cycles per unit of time). 


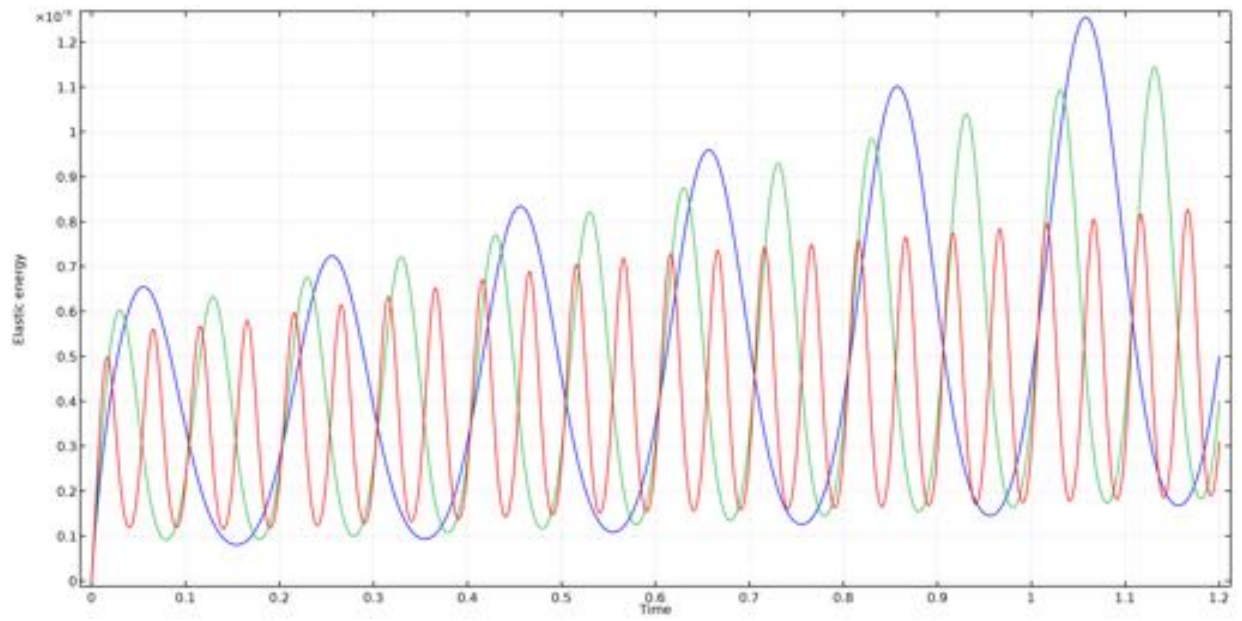

Figure 9: History of the elastic energy at the probe point $P_{m}$ for different load frequencies $(5,10$ and 20 cycles per unit of time).

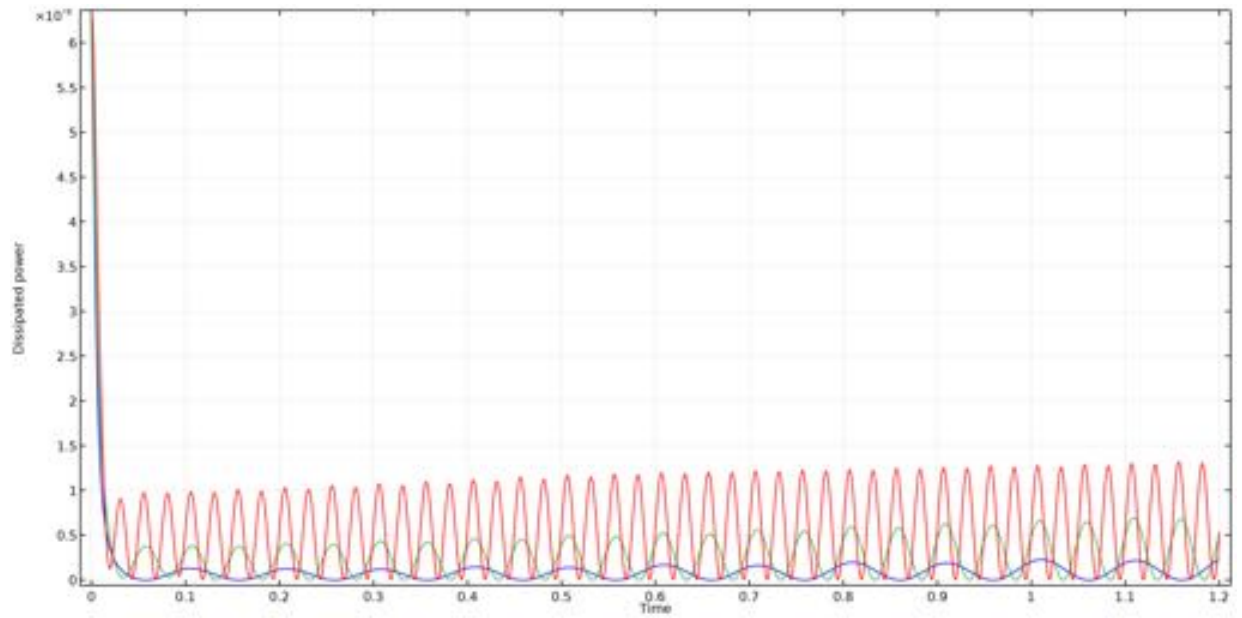

Figure 10: History of the dissipation at the probe point $P_{m}$ for different load frequencies $(5,10$ and 20 cycles per unit of time). 


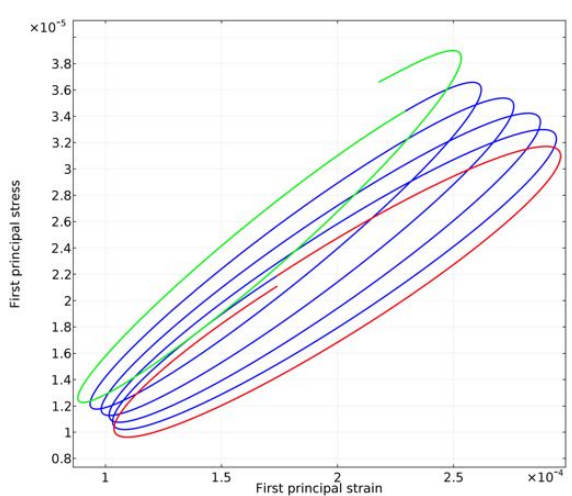

(a) freq. 5 cycles per unit of time

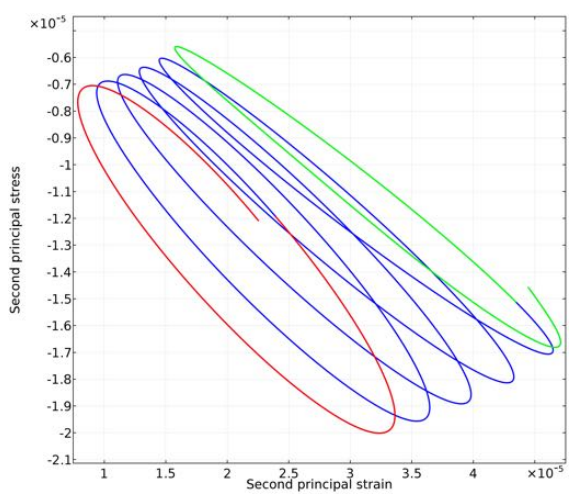

(d) freq. 5 cycles per unit of time

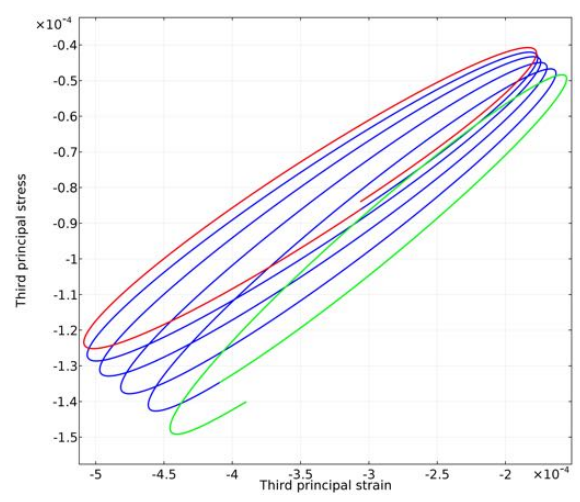

(g) freq. 5 cycles per unit of time

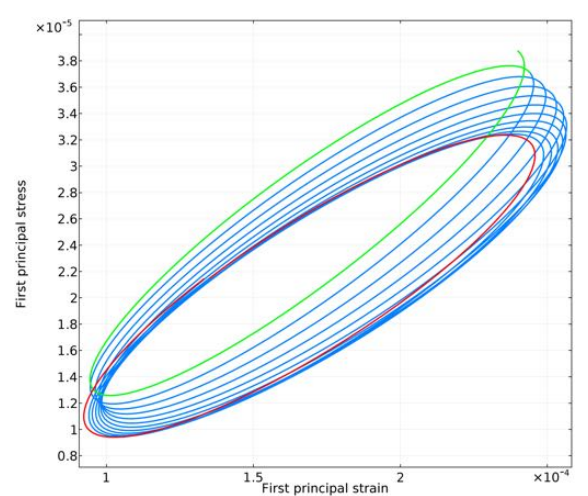

(b) freq. 10 cycles per unit of time

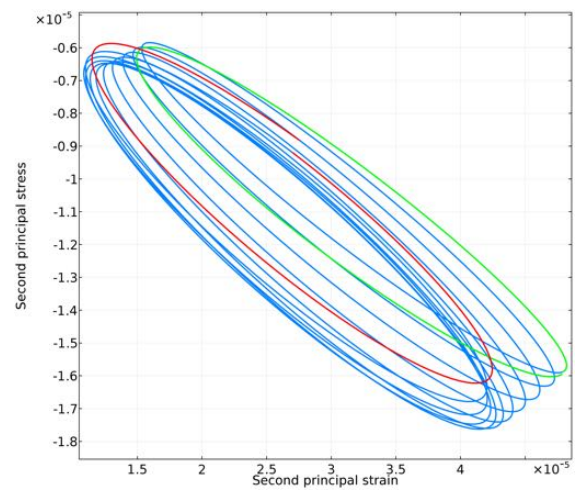

(e) freq. 10 cycles per unit of time

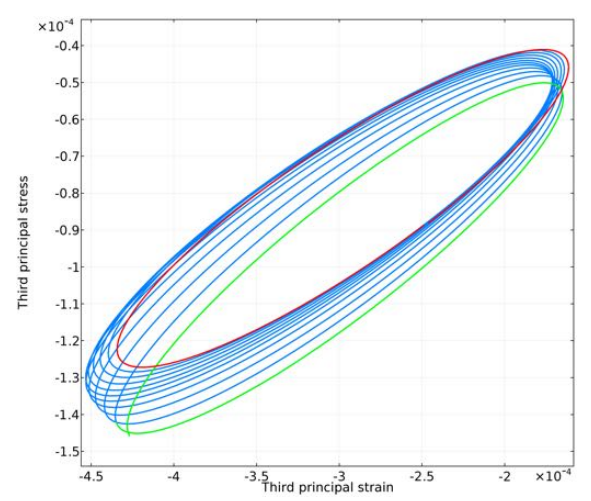

(h) freq. 10 cycles per unit of time

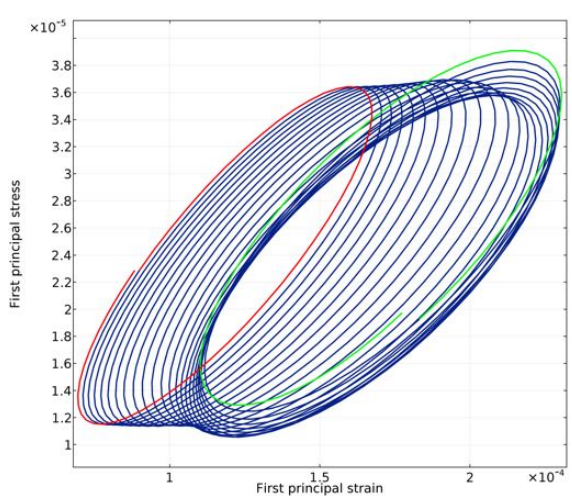

(c) freq. 20 cycles per unit of time

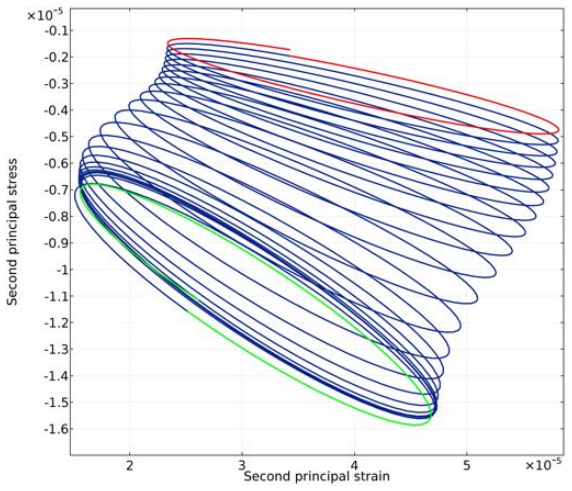

(f) freq. 20 cycles per unit of time

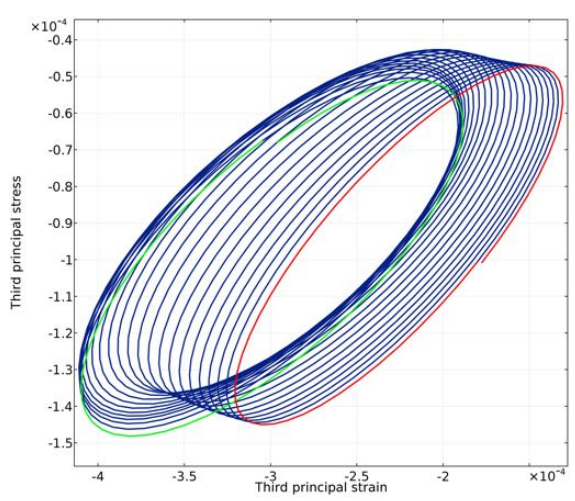

(i) freq. 20 cycles per unit of time

Figure 11: Energy dissipation loops for the three principal directions ((a),(b),(c) - first principal direction; (d),(e),(f) - second principal direction; $(\mathrm{g}),(\mathrm{h}),(\mathrm{i})$ - third principal direction) at the probe point $P_{c}$. 
whose coordinates are $\left(0.3 R_{e}, 1.154 R_{e}\right)$. The diagrams were obtained by plotting the principal stresses in terms of the corresponding principal strains for different frequencies. The rows correspond to the principal directions and the columns to the loading frequencies. The area subtended by each cycle is proportional to the energy dissipated in the same cycle. The first cycle is marked with green color, the last cycle with red color. The shape of each cycle is approximately elliptical, due to the dissipation of a viscous nature. The problem is complex, depending on such factors that influence each other resulting interdependent as the stimulus, the dissipation, the evolution of the mass density of bone material which in turn affects the stiffness of the mixture and thus the mechanical behavior. Starting from the third principal direction (third row), we note that both compressive stress and strain are negative. At low frequencies (5 cycles per unit of time), the deformation increases, the stress decreases and the final cycle is slightly larger than the initial one, that is, the dissipation grows. The growth process is slower; because less bone mass is produced and an erosion occurs in the area of the material (Fig. 4a) a higher porosity, a lower stiffness and a larger deformation are therefore attained. At high frequencies (20 cycles per unit of time), it is observed a different behavior compared to the one at low frequencies, by virtue of the faster evolution of bone mass density. In fact, deformation and dissipation significantly decrease, whereas a moderate stress reduction is exhibited. For intermediate frequencies, the two conflicting behaviors are approximately balanced and then final and initial cycles do not significantly differ in terms of both deformation and dissipation. Turning to the first principal direction (first row), characterized by deformation of elongation and tensile stress, it is observed a behavior similar to that already noted for the third principal direction. In fact, the factor which drives the described phenomenon is the rate of growth of the bone mass related to the frequency of the applied action. For low frequencies, deformation and dissipation increase while the stress decreases. For high frequencies, deformation, stress and dissipation decrease. For intermediate frequencies, the deformation and dissipation are approximately comparable. Finally, the second principal direction (second row) refers to the circumferential direction $\theta$. From a quantitative point of view, the deformations are of an order of magnitude smaller compared to the deformations in the other two principal directions (Poisson's effect), but the overall behavior reflects the considerations already made with regard to the two other principal directions.

Figure 12 shows the influence of the width of the lazy zone on the reference porosity $\phi^{*}$ of the mixture at the end of the process, at the frequency of 5 cycles per unit of time. The resorption threshold $\tilde{P}_{\text {ref }}^{\text {r has }}$ been fixed and the synthesis threshold $\tilde{P}_{\text {ref }}^{\mathrm{s}}$ is increased from the value equal to $\tilde{P}_{\text {ref }}^{\mathrm{r}}$. It is observed that as the synthesis threshold increases, the red area characterized by low porosity (high mass density) visibly decreases in the most stressed area below the implant. 


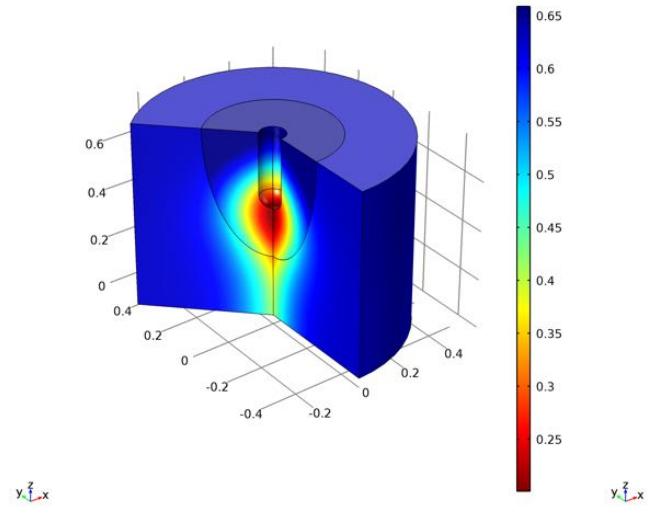

(a) $\tilde{P}_{\text {ref }}^{\mathrm{s}}=\tilde{P}_{\text {ref }}^{\mathrm{r}}=1.63 \times 10^{-9}$

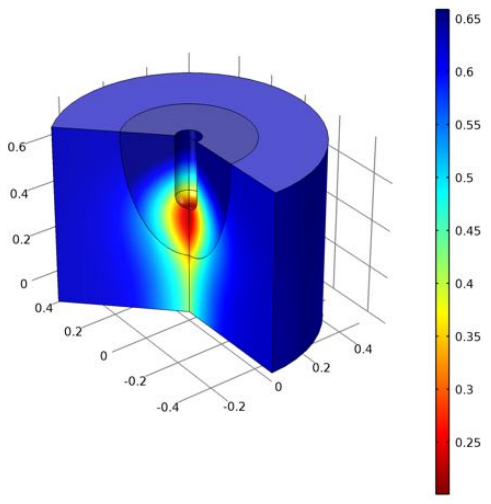

(b) $\tilde{P}_{\text {ref }}^{\mathrm{s}}=2.21 \times 10^{-9}$

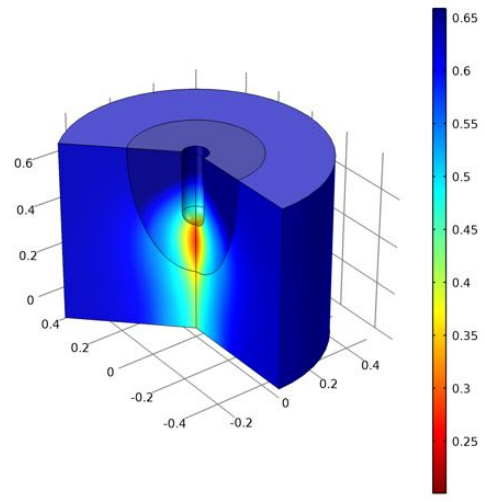

(c) $\tilde{P}_{\text {ref }}^{\mathrm{s}}=3.32 \times 10^{-9}$

Figure 12: Influence of the lazy zone: reference porosity $\phi^{*}$ at the end of the simulation and with a frequency load of 5 cycles per unit of time. 


\section{Conclusions}

The subject of this work is a type of dental implant IMZ that is suitable to be inserted in soft and atrophic bone tissue of mandibular jaw. It was studied the interaction of the implant with the biomaterial and the functional adaptation of the bone tissue under a load slowly variable in time with different frequencies, that produces a mechanical non-local stimulus consisting in the linear combination of deformation energy and viscous dissipation. The main mechanical parameters that have been considered are: mass density, porosity, change of porosity, density of strain energy and viscous dissipation. Attention was focused on the evaluation of the influence of the load frequency and of the amplitude of the lazy zone. The increase of frequency within a suitable range favors the production of bone as already reported in many studies (see e.g. $[75,76]$ ). However, if the stimulus is such to induce a too fast process of bone growth, then the conditions that should facilitate resorption of the biomaterial are not experienced. Therefore, the problem is to find a fair compromise between osteointegration and resorption of the biomaterial. The aim of this study was precisely to investigate the ways in which this compromise occurs and to quantify the limits of implementation. As regards the influence of the lazy zone, the performed numerical simulations have confirmed and quantified what might be expected intuitively, namely that the phenomenon of growth of the bone tissue is slowed down by an increase in the threshold of the stimulus. The numerical results presented in this study can be considered in view of reducing the risk of mobilization of the metallic stem with respect of the host bone tissue in the medium and long term; indeed, such a risk is due to the possibility of a non-perfect osteointegration at the interface between the implant cylinder and the jawbone.

\section{Acknowledgments}

The research was funded by the Italian Ministry of University and Research, under the Scientific Research Program of Relevant National Interest: Year 2010-2011, Protocol 2010MBJK5B-005, Title "Dynamics, Stability and Control of Flexible Structures".

\section{References}

[1] M. Soncini, R. Pietrabissa, and R. Rodriguez y Baena, "Computational approach for the mechanical reliability of a dental implant," in Computer Methods in Biomechanics and Biomedical Engineering (J. Middleton, G. N. Pande, and M. L. Jones, eds.), 2001.

[2] U. Andreaus, M. Colloca, and D. Iacoviello, "Coupling image processing and stress analysis for damage identification in a human premolar tooth," Computer methods and programs in biomedicine, vol. 103, no. 2, pp. 61-73, 2011.

[3] A. Ancillao and U. Andreaus, "Finite element analysis of the stress state produced by an orthodontic skeletal anchorage system based on miniscrews," Journal of Cranio-Maxillary Diseases, vol. 2, no. 1, p. $28,2013$. 
[4] O. Marangos, A. Misra, P. Spencer, B. Bohaty, and J. L. Katz, "Physico-mechanical properties determination using microscale homotopic measurements: application to sound and caries-affected primary tooth dentin," Acta biomaterialia, vol. 5, no. 4, pp. 1338-1348, 2009.

[5] G. A. Niznick, "Product profile-achieving osseointegration in soft bone: The search for improved results," Oral Health, vol. 90, no. 8, pp. 27-34, 2000.

[6] C. A. Babbush, A. Kirsch, P. J. Mentag, and B. Hill, "Intramobile cylinder (IMZ) two-stage osteointegrated implant system with the intramobile element (IME): part I. Its rationale and procedure for use.," The International journal of oral \&5 maxillofacial implants, vol. 2, no. 4, p. 203, 1987.

[7] G. Byrne, Fundamentals of Implant Dentistry. John Wiley \& Sons, 2014.

[8] A. Di Egidio, A. Luongo, and A. Paolone, "Linear and non-linear interactions between static and dynamic bifurcations of damped planar beams," International Journal of Non-Linear Mechanics, vol. 42 , no. 1 , pp. 88-98, 2007.

[9] A. Luongo, D. Zulli, and G. Piccardo, "On the effect of twist angle on nonlinear galloping of suspended cables," Computers \& Structures, vol. 87, no. 15, pp. 1003-1014, 2009.

[10] G. C. Ruta, V. Varano, M. Pignataro, and N. L. Rizzi, "A beam model for the flexural-torsional buckling of thin-walled members with some applications," Thin Wall. Struct., vol. 46, no. 7-9, pp. 816$822,2008$.

[11] N. L. Rizzi and V. Varano, "On the postbuckling analysis of thin-walled frames," 2011. DOI: 10.4203/ccp.96.43.

[12] N. Rizzi, V. Varano, and S. Gabriele, "Initial postbuckling behavior of thin-walled frames under mode interaction," Thin-Walled Structures, vol. 68, pp. 124-134, 2013.

[13] A. Grillo, S. Federico, G. Wittum, S. Imatani, G. Giaquinta, and M. V. Micunovic, "Evolution of a fibre-reinforced growing mixture," Il Nuovo cimento della Società italiana di fisica. C, vol. 32, no. 1, p. $97,2009$.

[14] L. Placidi, F. dell'Isola, N. Ianiro, and G. Sciarra, "Variational formulation of pre-stressed solid-fluid mixture theory, with an application to wave phenomena," European Journal of Mechanics-A/Solids, vol. 27 , no. 4 , pp. 582-606, 2008.

[15] L. Placidi and K. Hutter, "Thermodynamics of polycrystalline materials treated by the theory of mixtures with continuous diversity," Continuum Mechanics and Thermodynamics, vol. 17, no. 6, pp. 409-451, 2006.

[16] I. Goda, M. Assidi, S. Belouettar, and J.-F. Ganghoffer, "A micropolar anisotropic constitutive model of cancellous bone from discrete homogenization," Journal of the mechanical behavior of biomedical materials, vol. 16, pp. 87-108, 2012. 
[17] H. Altenbach and V. Eremeyev, "Analysis of the viscoelastic behavior of plates made of functionally graded materials," Zeitschrift für Angewandte Mathematik und Mechanik, vol. 88, no. 5, pp. 332-341, 2008.

[18] H. Altenbach, V. A. Eremeyev, L. P. Lebedev, and L. A. Rendón, "Acceleration waves and ellipticity in thermoelastic micropolar media," Archive of Applied Mechanics, vol. 80, no. 3, pp. 217-227, 2010.

[19] H. Aminpour and N. Rizzi, "A one-dimensional continuum with microstructure for single-wall carbon nanotubes bifurcation analysis," Mathematics and Mechanics of Solids, vol. 21, no. 2, pp. 168-181, 2016.

[20] S. C. Cowin and J. W. Nunziato, "Linear elastic materials with voids," Journal of Elasticity, vol. 13, no. 2, pp. 125-147, 1983.

[21] M. A. Biot, "Mechanics of deformation and acoustic propagation in porous media," Journal of applied physics, vol. 33, no. 4, pp. 1482-1498, 1962.

[22] M. A. Biot, "Generalized theory of acoustic propagation in porous dissipative media," The Journal of the Acoustical Society of America, vol. 34, no. 9A, pp. 1254-1264, 1962.

[23] S. C. Cowin, "Bone poroelasticity," Journal of biomechanics, vol. 32, no. 3, pp. 217-238, 1999.

[24] F. dell'Isola, L. Rosa, and C. Woźniak, "A micro-structured continuum modelling compacting fluidsaturated grounds: The effects of pore-size scale parameter," Acta mechanica, vol. 127, no. 1-4, pp. $165-182,1998$.

[25] F. dell'Isola and R. C. Batra, "Saint-Venant's problem for porous linear elastic materials," Journal of elasticity, vol. 47, no. 1, pp. 73-81, 1997.

[26] A. Madeo, F. dell'Isola, and F. Darve, "A continuum model for deformable, second gradient porous media partially saturated with compressible fluids," Journal of the Mechanics and Physics of Solids, vol. 61, no. 11, pp. 2196-2211, 2013.

[27] A. Madeo, F. dell'Isola, N. Ianiro, and G. Sciarra, "A variational deduction of second gradient poroelasticity II: An application to the consolidation problem," Journal of Mechanics of Materials and Structures, vol. 3, no. 4, pp. 607-625, 2008.

[28] A. Tomic, A. Grillo, and S. Federico, "Poroelastic materials reinforced by statistically oriented fibresnumerical implementation and application to articular cartilage," IMA Journal of Applied Mathematics, vol. 79, no. 5, pp. 1027-1059, 2014.

[29] S. Federico, A. Grillo, W. Herzog, G. Giaquinta, and S. Imatani, "Possible approaches in modelling rearrangement in a microstructured material," in Key Engineering Materials, vol. 340, pp. 137-142, Trans Tech Publ, 2007. 
[30] A. Grillo, R. Prohl, and G. Wittum, "A poroplastic model of structural reorganisation in porous media of biomechanical interest," Continuum Mechanics and Thermodynamics, vol. 28, no. 1, pp. 579-601, 2016.

[31] F. dell'Isola, L. Rosa, and C. Woźniak, "Dynamics of solids with microperiodic nonconnected fluid inclusions," Archive of Applied Mechanics, vol. 67, pp. 215-228, 1997.

[32] J.-F. Ganghoffer, "A contribution to the mechanics and thermodynamics of surface growth. Application to bone external remodeling," International Journal of Engineering Science, vol. 50, no. 1, pp. 166-191, 2012.

[33] J.-F. Ganghoffer, "Mechanical modeling of growth considering domain variation-part II: Volumetric and surface growth involving eshelby tensors," Journal of the Mechanics and Physics of Solids, vol. 58, no. 9, pp. 1434-1459, 2010.

[34] L. Placidi, S. H. Faria, and K. Hutter, "On the role of grain growth, recrystallization and polygonization in a continuum theory for anisotropic ice sheets," Annals of Glaciology, vol. 39, no. 1, pp. 49-52, 2004.

[35] L. Placidi and K. Hutter, "An anisotropic flow law for incompressible polycrystalline materials," Zeitschrift für angewandte Mathematik und Physik ZAMP, vol. 57, no. 1, pp. 160-181, 2005.

[36] F. dell'Isola and K. Hutter, "What are the dominant thermomechanical processes in the basal sediment layer of large ice sheets?," Proceedings of the Royal Society of London A: Mathematical, Physical and Engineering Sciences, vol. 454, no. 1972, pp. 1169-1195, 1998.

[37] F. dell'Isola and A. Romano, "A phenomenological approach to phase transition in classical field theory," International journal of engineering science, vol. 25, no. 11, pp. 1469-1475, 1987.

[38] F. dell'Isola and C. Woźniak, "On phase transition layers in certain micro-damaged two-phase solids," International Journal of Fracture, vol. 83, no. 2, pp. 175-189, 1997.

[39] V. A. Yeremeyev, A. B. Freidin, and L. L. Sharipova, "The stability of the equilibrium of two-phase elastic solids," Journal of applied mathematics and mechanics, vol. 71, no. 1, pp. 61-84, 2007.

[40] V. A. Eremeyev and W. Pietraszkiewicz, "Phase transitions in thermoelastic and thermoviscoelastic shells," Archives of Mechanics, vol. 61, no. 1, pp. 41-67, 2009.

[41] U. Andreaus, I. Giorgio, and A. Madeo, "Modeling of the interaction between bone tissue and resorbable biomaterial as linear elastic materials with voids," Zeitschrift für angewandte Mathematik und Physik, vol. 66, no. 1, pp. 209-237, 2015.

[42] P. Seppecher, "Second-gradient theory: application to Cahn-Hilliard fluids," in Continuum Thermomechanics, pp. 379-388, Springer, 2002. 
[43] Y. Yang and A. Misra, "Higher-order stress-strain theory for damage modeling implemented in an element-free galerkin formulation," Computer Modeling in Engineering 6 Sciences, vol. 64, no. 1, pp. 1-36, 2010.

[44] Y. Yang, W. Y. Ching, and A. Misra, "Higher-order continuum theory applied to fracture simulation of nanoscale intergranular glassy film," Journal of Nanomechanics and Micromechanics, vol. 1, no. 2, pp. 60-71, 2011.

[45] L. Placidi, "A variational approach for a nonlinear 1-dimensional second gradient continuum damage model," Continuum Mechanics and Thermodynamics, vol. 27, no. 4-5, pp. 623-638, 2015.

[46] L. Placidi, "A variational approach for a nonlinear one-dimensional damage-elasto-plastic secondgradient continuum model," Continuum Mechanics and Thermodynamics, vol. 28, pp. 119-137, 2016.

[47] A. Rinaldi and L. Placidi, "A microscale second gradient approximation of the damage parameter of quasi-brittle heterogeneous lattices," ZAMM-Journal of Applied Mathematics and Mechanics/Zeitschrift für Angewandte Mathematik und Mechanik, vol. 94, no. 10, pp. 862-877, 2014.

[48] U. Andreaus, G. Ceradini, P. D'Asdia, and P. Gaudenzi, "Damage modelling and seismic response of simple degrading systems," Res mechanica, vol. 22, no. 1, pp. 79-100, 1987.

[49] A. Carcaterra, F. dell'Isola, R. Esposito, and M. Pulvirenti, "Macroscopic description of microscopically strongly inhomogenous systems: A mathematical basis for the synthesis of higher gradients metamaterials," Archive for Rational Mechanics and Analysis, vol. 218, no. 3, pp. 1239-1262, 2015.

[50] T. C. Lee, A. Staines, and D. Taylor, "Bone adaptation to load: microdamage as a stimulus for bone remodelling," Journal of Anatomy, vol. 201, no. 6, pp. 437-446, 2002.

[51] E. D. Sedlin, "A rheologic model for cortical bone: a study of the physical properties of human femoral samples," Acta Orthopaedica, vol. 36, no. S83, pp. 1-77, 1965.

[52] J. H. Bargren, C. A. L. Bassett, and A. Gjelsvik, "Mechanical properties of hydrated cortical bone," Journal of biomechanics, vol. 7, no. 3, pp. 239-245, 1974.

[53] A. Carcaterra and A. Akay, "Dissipation in a finite-size bath," Physical Review E, vol. 84, no. 1, p. 011121, 2011.

[54] A. Carcaterra, N. Roveri, and G. Pepe, "Fractional dissipation generated by hidden wave-fields," Mathematics and Mechanics of Solids, vol. 20, no. 10, pp. 1251-1262, 2015.

[55] T. Lekszycki, "Application of variational methods in analysis and synthesis of viscoelastic continuous systems," J Struct Mech, vol. 19, no. 2, pp. 163-192, 1991.

[56] F. dell'Isola, A. Madeo, and P. Seppecher, "Cauchy tetrahedron argument applied to higher contact interactions," Archive for Rational Mechanics and Analysis, vol. 219, no. 3, pp. 1305-1341, 2016. 
[57] F. dell'Isola, P. Seppecher, and A. Della Corte, "The postulations á la D'Alembert and á la Cauchy for higher gradient continuum theories are equivalent: a review of existing results," Proceedings of the Royal Society of London A, vol. 471, no. 2183, p. 20150415, 2015.

[58] I. Giorgio, U. Andreaus, D. Scerrato, and F. dell'Isola, "A visco-poroelastic model of functional adaptation in bones reconstructed with bio-resorbable materials," Biomechanics and Modeling in Mechanobiology, 2016. DOI: 10.1007/s10237-016-0765-6.

[59] T. Lekszycki and F. dell'Isola, "A mixture model with evolving mass densities for describing synthesis and resorption phenomena in bones reconstructed with bio-resorbable materials," ZAMM-Journal of Applied Mathematics and Mechanics/Zeitschrift für Angewandte Mathematik und Mechanik, vol. 92, no. 6, pp. 426-444, 2012.

[60] M. G. Mullender and R. Huiskes, "Proposal for the regulatory mechanism of wolff's law," Journal of orthopaedic research, vol. 13, no. 4, pp. 503-512, 1995.

[61] A. Madeo, T. Lekszycki, and F. dell'Isola, "A continuum model for the bio-mechanical interactions between living tissue and bio-resorbable graft after bone reconstructive surgery," Comptes Rendus Mécanique, vol. 339, no. 10, pp. 625-640, 2011.

[62] C. Kumar, I. Jasiuk, and J. Dantzig, "Dissipation energy as a stimulus for cortical bone adaptation," Journal of Mechanics of Materials and Structures, vol. 6, no. 1, pp. 303-319, 2011.

[63] C. H. Turner, "Homeostatic control of bone structure: an application of feedback theory," Bone, vol. 12 , no. 3, pp. 203-217, 1991.

[64] H. Weinans, R. Huiskes, and H. J. Grootenboer, "The behavior of adaptive bone-remodeling simulation models," Journal of biomechanics, vol. 25, no. 12, pp. 1425-1441, 1992.

[65] R. Hambli, "Connecting mechanics and bone cell activities in the bone remodeling process: an integrated finite element modeling," Frontiers in bioengineering and biotechnology, 2014. DOI: 10.3389/fbioe.2014.00006.

[66] S. C. Cowin and D. H. Hegedus, "Bone remodeling I: theory of adaptive elasticity," Journal of Elasticity, vol. 6, no. 3, pp. 313-326, 1976.

[67] D. R. Carter and W. E. Caler, "A cumulative damage model for bone fracture," Journal of Orthopaedic Research, vol. 3, no. 1, pp. 84-90, 1985.

[68] W. E. Caler and D. R. Carter, "Bone creep-fatigue damage accumulation," Journal of Biomechanics, vol. 22 , no. 6 , pp. $625-635,1989$.

[69] T. Lekszycki, "Modelling of bone adaptation based on an optimal response hypothesis," Meccanica, vol. 37, no. 4-5, pp. 343-354, 2002. 
[70] I. G. Jang and I. Y. Kim, "Application of design space optimization to bone remodeling simulation of trabecular architecture in human proximal femur for higher computational efficiency," Finite Elements in Analysis and Design, vol. 46, no. 4, pp. 311-319, 2010.

[71] U. Andreaus, M. Colloca, D. Iacoviello, and M. Pignataro, "Optimal-tuning pid control of adaptive materials for structural efficiency," Structural and Multidisciplinary Optimization, vol. 43, no. 1, pp. 43-59, 2011.

[72] U. Andreaus, M. Colloca, and D. Iacoviello, "An optimal control procedure for bone adaptation under mechanical stimulus," Control Engineering Practice, vol. 20, no. 6, pp. 575-583, 2012.

[73] U. Andreaus, M. Colloca, and D. Iacoviello, "Modeling of trabecular architecture as result of an optimal control procedure," in Biomedical Imaging and Computational Modeling in Biomechanics (D. Iacoviello and U. Andreaus, eds.), ch. II, pp. 19-37, Dordrecht, Springer, 2013.

[74] U. Andreaus, M. Colloca, and D. Iacoviello, "Optimal bone density distributions: Numerical analysis of the osteocyte spatial influence in bone remodeling," Computer methods and programs in biomedicine, vol. 113, no. 1, pp. 80-91, 2014.

[75] L. E. Lanyon and C. T. Rubin, "Static vs dynamic loads as an influence on bone remodelling," Journal of biomechanics, vol. 17, no. 12, pp. 897-905, 1984.

[76] C. H. Turner, "Three rules for bone adaptation to mechanical stimuli," Bone, vol. 23, no. 5, pp. 399407, 1998.

[77] U. Andreaus, I. Giorgio, and T. Lekszycki, "A 2-D continuum model of a mixture of bone tissue and bio-resorbable material for simulating mass density redistribution under load slowly variable in time," Zeitschrift für Angewandte Mathematik und Mechanik, vol. 94, no. 12, pp. 978-1000, 2014.

[78] I. Giorgio, U. Andreaus, and A. Madeo, "The influence of different loads on the remodeling process of a bone and bioresorbable material mixture with voids," Continuum Mechanics and Thermodynamics, vol. 28 , no. 1, pp. 21-40, 2016.

[79] I. Giorgio, L. Galantucci, A. Della Corte, and D. Del Vescovo, "Piezo-electromechanical smart materials with distributed arrays of piezoelectric transducers: current and upcoming applications," International Journal of Applied Electromagnetics and Mechanics, vol. 47, no. 4, pp. 1051-1084, 2015.

[80] I. Giorgio, A. Culla, and D. Del Vescovo, "Multimode vibration control using several piezoelectric transducers shunted with a multiterminal network," Archive of Applied Mechanics, vol. 79, no. 9, pp. 859-879, 2009.

[81] M. Porfiri, F. dell'Isola, and E. Santini, "Modeling and design of passive electric networks interconnecting piezoelectric transducers for distributed vibration control," International Journal of Applied Electromagnetics and Mechanics, vol. 21, no. 2, pp. 69-87, 2005. 
[82] C. Maurini, F. dell'Isola, and D. Del Vescovo, "Comparison of piezoelectronic networks acting as distributed vibration absorbers," Mechanical Systems and Signal Processing, vol. 18, no. 5, pp. 12431271, 2004.

[83] L. C. Pagnini and G. Piccardo, "The three-hinged arch as an example of piezomechanic passive controlled structure," Continuum Mechanics and Thermodynamics, 2015. DOI: 10.1007/s00161-0150474-x.

[84] D. Del Vescovo and I. Giorgio, "Dynamic problems for metamaterials: review of existing models and ideas for further research," International Journal of Engineering Science, vol. 80, pp. 153-172, 2014.

[85] F. dell'Isola, T. Lekszycki, M. Pawlikowski, R. Grygoruk, and L. Greco, "Designing a light fabric metamaterial being highly macroscopically tough under directional extension: first experimental evidence," Zeitschrift für angewandte Mathematik und Physik, vol. 66, no. 6, pp. 3473-3498, 2015.

[86] F. dell'Isola, A. Della Corte, L. Greco, and A. Luongo, "Plane bias extension test for a continuum with two inextensible families of fibers: a variational treatment with Lagrange multipliers and a perturbation solution," International Journal of Solids and Structures, vol. 81, pp. 1-12, 2016.

[87] J.-J. Alibert and A. Della Corte, "Second-gradient continua as homogenized limit of pantographic microstructured plates: a rigorous proof," Zeitschrift für angewandte Mathematik und Physik, vol. 66, no. 5, pp. 2855-2870, 2015.

[88] A. Madeo, A. Della Corte, L. Greco, and P. Neff, "Wave propagation in pantographic 2D lattices with internal discontinuities," Proceedings of the Estonian Academy of Sciences, vol. 64, no. 3S, pp. 325-330, 2015 .

[89] A. Cazzani, M. Malagù, and E. Turco, "Isogeometric analysis of plane-curved beams," Mathematics and Mechanics of Solids, 2014. DOI: 10.1177/1081286514531265.

[90] E. Turco and M. Aristodemo, "A three-dimensional b-spline boundary element," Computer methods in applied mechanics and engineering, vol. 155, no. 1, pp. 119-128, 1998.

[91] A. Cazzani, M. Malagù, and E. Turco, "Isogeometric analysis: a powerful numerical tool for the elastic analysis of historical masonry arches," Continuum Mechanics and Thermodynamics, vol. 28, no. 1, pp. 139-156, 2016.

[92] A. Cazzani, M. Malagù, E. Turco, and F. Stochino, "Constitutive models for strongly curved beams in the frame of isogeometric analysis," Mathematics and Mechanics of Solids, vol. 21, no. 2, pp. 182-209, 2016.

[93] A. Della Corte, A. Battista, and F. dell'Isola, "Referential description of the evolution of a 2D swarm of robots interacting with the closer neighbors: Perspectives of continuum modeling via higher gradient continua," International Journal of Non-Linear Mechanics, vol. 80, no. 209-220, 2016. 
[94] S. Federico, A. Grillo, S. Imatani, G. Giaquinta, and W. Herzog, "An energetic approach to the analysis of anisotropic hyperelastic materials," International Journal of Engineering Science, vol. 46, no. 2, pp. 164-181, 2008.

[95] G. Solari, L. C. Pagnini, and G. Piccardo, "A numerical algorithm for the aerodynamic identification of structures," Journal of wind engineering and industrial aerodynamics, vol. 69, pp. 719-730, 1997.

[96] L. Greco and M. Cuomo, "B-Spline interpolation of Kirchhoff-Love space rods," Computer Methods in Applied Mechanics and Engineering, vol. 256, pp. 251-269, 2013.

[97] L. Greco and M. Cuomo, "An implicit G1 multi patch B-spline interpolation for Kirchhoff-Love space rod," Computer Methods in Applied Mechanics and Engineering, vol. 269, pp. 173-197, 2014.

[98] L. Greco and M. Cuomo, "An isogeometric implicit G1 mixed finite element for Kirchhoff space rods," Comput Methods Appl Mech Eng, vol. 298, pp. 325-349, 2016.

[99] M. Cuomo, L. Contrafatto, and L. Greco, "A variational model based on isogeometric interpolation for the analysis of cracked bodies," International Journal of Engineering Science, vol. 80, pp. 173-188, 2014. 